\title{
A floating potential method for measuring ion density
}

Francis F. Chen, John D. Evans, and Donald Arnush

Electrical Engineering Department, University of California Los Angeles

Los Angeles, California 90095-1594

\begin{abstract}
A simple method for analyzing Langmuir probe curves is described which yields the ion density in weakly collisional plasmas for which purely collisionless theories give erroneous results. The method is based on an extrapolation to the floating potential of the saturation ion current raised to the $4 / 3$ power. This procedure is not supported by theory but apparently works because effects neglected in the theory tend to cancel.
\end{abstract}

\section{INTRODUCTION AND METHODOLOGY}

Langmuir probe measurement of the plasma density $n$ in the weakly collisional radiofrequency (rf) discharges used in semiconductor fabrication is difficult not only because of contamination of the probe tips but also because it has been found ${ }^{1}$ that collisionless theories of ion collection are subject to large errors in these plasmas. We have found experimentally that saturation ion currents $I_{\mathrm{i}}$ to cylindrical probes in the $10^{10}$ to $10^{12} \mathrm{~cm}^{-3}$ density range tend to follow an $I_{\mathrm{i}} \propto V_{\mathrm{p}}^{3 / 4}$ law. Extrapolating to the floating potential $V_{\mathrm{f}}$, which is easily measured, one can obtain an estimate of the ion (or electron) current at $V_{\mathrm{f}}$. Since $V_{\mathrm{f}} \approx$ $5 K T_{\mathrm{e}}$, the sheath is well established at this potential, and the expected $I_{\mathrm{i}}\left(V_{\mathrm{f}}\right)$ can be calculated without the uncertainties inherent in extrapolating to the space potential $V_{\mathrm{s}}(\equiv 0)$ due to the weak ion-accelerating fields there. The $I_{\mathrm{i}} \propto V_{\mathrm{p}}^{3 / 4}$ is reminiscent of the Child-Langmuir (CL) law for plane electrodes. If one assumes that the sheath thickness is given by the CL law (neglecting the cylindrical curvature), the collection area expands as $V_{\mathrm{p}}^{3 / 4}$, giving rise to the observed shape of the $I-V$ curve. The ion current at the sheath edge is given by the Bohm sheath criterion as

$$
I_{i}=\alpha_{0} n A_{s} c_{s}, \quad c_{S} \equiv\left(K T_{e} / M\right)^{1 / 2},
$$

where $A_{\mathrm{S}}$ is the sheath area, and $\alpha_{0}$ is a constant equal to $\mathrm{e}^{-1 / 2}=0.61$ if $T_{\mathrm{i}}=0$ and $\approx 0.5$ if $T_{\mathrm{i}}$ is slightly elevated above room temperature ${ }^{2}$. Hence, knowing $V_{\mathrm{f}}-V_{\mathrm{s}}$ and $I_{\mathrm{i}}\left(V_{\mathrm{f}}\right)$, one can compute $n$ using the value of $K T_{\mathrm{e}}$ from the electron part of the $I-V$ curve. As we shall show in detail, the CL formula should not be applicable in these circumstances, but this procedure heuristically gives values of $n$ in good agreement with independent measurements using microwave interferometry. [Note that $I_{\mathrm{i}}$ is a particle current; the electrical current is $e I_{\mathrm{i}}$, the ions being assumed singly charged.]

The procedure, then, is as follows. A sample $I-V$ curve is shown in Fig. 1. First, the space potential $V_{\mathrm{s}}$ is found from the minimum in the $d I / d V$ curve, shown in Fig. 2 . In this case, a clear minimum can be found by drawing a smooth curve through the data points. In RF discharges plasma noise and inadequate RF compensation often make this curve hashy, sometimes with more than one minimum, as illustrated in Fig. 3 for a different $I-V$ curve. The experimenter has then to choose $V_{\mathrm{s}}$ judiciously. Next, the ion part of the characteristic is 
raised to the $4 / 3$ power and plotted against $V_{\mathrm{p}}$, as shown in Fig. 4. A straight line is fitted to the part of the curve that is not affected by electron current. Extrapolating to $V_{\mathrm{f}}$, where $I=I_{\mathrm{i}}$ $-I_{\mathrm{e}}=0$, gives an estimate of $I_{\mathrm{i}}\left(V_{\mathrm{f}}\right)$. Note that the extrapolation to $V_{\mathrm{f}}$ is much shorter than to $V_{\mathrm{s}}$, so that the estimate of $I_{\mathrm{i}}\left(V_{\mathrm{f}}\right)$ should be more accurate than $I_{\mathrm{i}}\left(V_{\mathrm{s}}\right)$. The ion current is then calculated from the straight line fit to $I_{\mathrm{i}}^{4 / 3}$ and subtracted from the total current to give $I_{\mathrm{e}}$. This is plotted semi-logarithmically in Fig. 5. Note that the ion correction to $I_{\mathrm{e}}$ has made the curve follow a Maxwellian over a much larger range of $V\left(=V_{\mathrm{p}}\right)$. Fitting a straight line to this curve yields the electron temperature $K T_{\mathrm{e}}$ in $\mathrm{eV}$ :

$$
I_{e}=A_{p} n v_{e r} \exp \left(-\eta_{p}\right), \quad v_{e r} \equiv\left(K T_{e} / 2 \pi m\right)^{1 / 2}, \quad \eta \equiv-\left(V-V_{s}\right) / K T_{e},
$$

$A_{\mathrm{p}}$ being the probe area and $n$ the plasma density in the body of the plasma. The reciprocal of the slope of the $\ln I_{\mathrm{e}}-V_{\mathrm{p}}$ curve is then equal to $K T_{\mathrm{e}}$. The value of $I_{\mathrm{e}}$ at $V_{\mathrm{p}}=V_{\mathrm{s}}$ gives an estimate of $n$, called $N_{\mathrm{e}}$, which is based on $I_{\mathrm{e}}$ alone. Since $N_{\mathrm{e}}$ depends exponentially on $V_{\mathrm{s}}$, it is subject to large errors arising from uncertainty in the determination of $V_{\mathrm{s}}$.

To find $n$ from $I_{\mathrm{i}}\left(V_{\mathrm{f}}\right)$, we assume a sheath thickness $d$ given by the CL formula ${ }^{3}$

$$
d=\frac{1}{3} \sqrt{\frac{2}{\alpha_{0}}}\left(2 \eta_{f}\right)^{3 / 4} \lambda_{D}=1.018 \eta_{f}^{3 / 4} \lambda_{D}, \quad \lambda_{D} \equiv\left(\varepsilon_{0} K T_{e} / n e^{2}\right)^{1 / 2}
$$

with $\eta_{\mathrm{f}}$ known once $V_{\mathrm{s}}$ and $T_{\mathrm{e}}$ have been determined. The sheath radius is the sum of $d$ and the probe radius $R_{\mathrm{p}}$. Using Eq. (3) in Eq. (1) for a probe length $L$ then gives

$$
n=N_{i}(C L)=I_{i}\left(V_{f}\right) / 2 \pi\left(R_{p}+d\right) L \alpha_{0} c_{s}
$$

Note, however, that $d$ depends on $\lambda_{\mathrm{D}}$, which is proportional to $n^{-1 / 2}$. Eqs. (3) and (4) thus constitute a quadratic equation for $n^{1 / 2}$, whose gives the ion density as

$$
n=\left\{\left[-B+\left(B^{2}+4 A C\right)^{1 / 2}\right] / 2 A\right\}^{2}
$$

where

$$
A=R_{p}, \quad B \approx \eta_{f}^{3 / 4}\left(\varepsilon_{0} K T_{e} / e^{2}\right)^{1 / 2}, \quad C=I_{i}\left(V_{f}\right) / 2 \pi L \alpha_{0} c_{s}
$$

\section{COMPARISON WITH THEORY}

Although it will be shown that this method yields values of $n$ in agreement with microwave measurements, it is not easy to justify theoretically. First, the CL formula used for $d$ is for plane sheaths, not cylindrical ones. Second, the CL formula gives only a crude approximation to the sheath thickness because it neglects the Debye sheath, where the electron density cannot be neglected. Treatments which jump discontinuously from quasineutral plasma to pure ion sheaths are referred to as step models ${ }^{4}$. Sometimes, a constant ion density is assumed is what is called a matrix sheath ${ }^{3}$. The Bohm formula requires ions to enter the sheath with the velocity $c_{\mathrm{s}}$, whereas the CL formula assumes zero velocity. Hutchinson ${ }^{5}$ has modified Eq. (3) to include an initial ion velocity $c_{\mathrm{s}}$. If we take $V=0$ in the plasma rather than at the sheath edge as in Ref. 5, Hutchinson's formula becomes

$$
d=1.018\left([\eta-1 / 2]^{1 / 2}-2^{-1 / 2}\right)^{1 / 2}\left([\eta-1 / 2]^{1 / 2}+2^{1 / 2}\right) \lambda_{D} .
$$

Use of this formula did not improve the results. 
The exact solution for a combined Debye-CL sheath in plane geometry can be derived from Poisson's equation

$$
\frac{d^{2} V}{d x^{2}}=\frac{e}{\varepsilon_{0}}\left(n_{e}-n_{i}\right)
$$

In a strictly 1-D problem, we must assume a sheath edge, since if the ion velocity $v_{\mathrm{i}}$ were zero at infinity, the density there would have to be infinite for the ion flux to be finite. For convenience we choose the sheath edge to be the plane where (a) $\mathrm{eV}=-1 / 2 K T_{\mathrm{e}}$ relative to the plasma potential, so that the Bohm criterion is satisfied there, (b) $v_{\mathrm{i}}=c_{\mathrm{s}}$, and (c) $n=n_{\mathrm{s}}=$ $n_{0} \mathrm{e}^{-1 / 2}$. Shifting the origin of both $x$ and $V$ to this point, we now have, for Maxwellian electrons,

$$
n_{e}=n_{s} e^{e V / K T_{e}}=n_{s} e^{-\eta}, \quad \eta \equiv-e V / K T_{e}
$$

For the ions, energy conservation requires

$$
1 / 2 M v_{i}^{2}=1 / 2 M c_{S}^{2}-e V, \quad v_{i}=\left(c_{S}^{2}-2 e V / M\right)^{1 / 2} .
$$

Continuity of ion flux then gives

$$
n_{i} v_{i}=n_{S} c_{s}, \quad n_{i}=n_{S} \frac{c_{S}}{v_{i}}=n_{S}\left(1-\frac{2 e V}{M c_{S}^{2}}\right)^{-1 / 2}=n_{s}(1+2 \eta)^{-1 / 2}
$$

Eq. (8) then becomes

$$
\frac{d^{2} V}{d x^{2}}=\frac{e}{\varepsilon_{0}} n_{s}\left[e^{-\eta}-(1+2 \eta)^{-1 / 2}\right]=-\frac{K T_{e}}{e} \frac{d^{2} \eta}{d x^{2}}
$$

Normalization to the Debye length $\lambda_{\mathrm{Ds}}$ at the sheath edge yields

$$
\eta^{\prime \prime}=\left[(1+2 \eta)^{-1 / 2}-e^{-\eta}\right]
$$

where the (') indicates derivative with respect to $\xi \equiv x / \lambda_{D s}$. Following standard procedure, we multiply by an integration factor $\eta^{\prime}$ and integrate from $\eta=0$ to $\eta$. Setting $\eta^{\prime}=0$ at $\xi=0$, we obtain

$$
\eta^{\prime}=\sqrt{2}\left[(1+2 \eta)^{1 / 2}+e^{-\eta}-2\right]^{1 / 2}
$$

The next integration has to be done numerically, and the result is shown in Fig. 6 for two assumed values of $\eta^{\prime \prime}(0)$. The "sheath thickness" is indeterminate because it depends on the assumed boundary condition at $\xi=0$. If $\eta^{\prime}=0$ there, then $\eta^{\prime \prime}$ or another derivative must be given a finite value in order for the curve to rise above zero. In practice, the boundary condition on $\eta^{\prime}$ and $\eta^{\prime \prime}$ is determined by matching to the presheath. The two curves of Fig. 6 are plotted logarithmically in Fig. 7. Once $\eta$ becomes appreciable, the curve $\eta(\xi)$ has a definite shape, but its position relative to the sheath edge cannot be found without a presheath calculation. Also shown in Fig. 7 is the CL sheath thickness according to Eq. (3) and the floating 
potential $\eta_{\mathrm{f}}=4.68$ of a plane probe ${ }^{6}$. We see that it is possible to choose a boundary condition that makes the computed sheath thickness agree with $d_{\mathrm{CL}}\left(V_{\mathrm{f}}\right)$, but that the slopes of the curves are different there. The increase in $I_{\mathrm{i}}$ with $V_{\mathrm{p}}$ depends on the normalized probe radius

$$
\xi_{p} \equiv R_{p} / \lambda_{D}
$$

Adding $\xi_{\mathrm{p}}$ to the right-hand curve of Fig. 7 results in Fig. 8. Although the sheath radius at $V_{\mathrm{f}}$ can agree with the CL prediction if the small gradients at the sheath edge happen to have the right value, the slopes do not agree at larger probe biases. Thus, it is difficult to see why the measured ion saturation current increases as $\left|V_{\mathrm{p}}\right|^{3 / 4}$, as predicted by the inexact CL formula but not by the exact calculation.

Since the use of the CL formula is not justified because the sheath is not plane by cylindrical, we next solve the sheath equation for cylinders. In this case, the calculation can be carried out to infinity, and the arbitrary conditions at a sheath edge are not needed. However, in a simple treatment, collisions are ignored as well as the orbiting of ions around the probe due to their angular momentum. Poisson's equation is now

$$
\frac{1}{r} \frac{\partial}{\partial r}\left(r \frac{\partial V}{\partial r}\right)=\frac{e}{\varepsilon_{0}}\left(n_{e}-n_{i}\right), \quad n_{e}=n_{0} e^{e V / K T_{e}}=n_{0} e^{-\eta}
$$

where $n \rightarrow n_{0}$ and $V \rightarrow 0$ as $r \rightarrow \infty$. The ion velocity is

$$
v_{i}=(-2 e V / M)^{1 / 2}=(2 \eta)^{1 / 2} c_{S},
$$

and flux conservation gives

$$
n_{i}=\frac{I_{i} / v_{i}}{2 \pi r}=\frac{I_{i}}{2 \pi r c_{S}}(2 \eta)^{-1 / 2}
$$

where $I_{\mathrm{i}}$ is the inward ion flux per unit probe length. Normalizing to $\rho \equiv r / \lambda_{\mathrm{D}}$, we now have for Eq. (16)

$$
\begin{aligned}
\frac{\partial}{\partial \rho}\left(\rho \frac{\partial \eta}{\partial \rho}\right) & =\frac{I_{i} \rho}{2 \pi r} \frac{1}{n_{0} c_{S}}(2 \eta)^{-1 / 2}-\rho e^{-\eta} \\
& =\frac{I_{i}}{2 \pi n_{0}} \frac{1}{\lambda_{D} c_{S}}(2 \eta)^{-1 / 2}-\rho e^{-\eta}
\end{aligned}
$$

The coefficient of the ion term is a dimensionless ion current defined by

$$
J \equiv \frac{I_{i}}{2^{3 / 2} \pi n_{0}} \frac{1}{\lambda_{D^{c}}}
$$

In terms of $J$, Poisson's equation is simply

$$
\frac{\partial}{\partial \rho}\left(\rho \frac{\partial \eta}{\partial \rho}\right)=J \eta^{-1 / 2}-\rho e^{-\eta}
$$

This is the cylindrical equivalent of the Allen-Boyd-Reynolds (ABR) equation for spherical probes $^{7}$. Solutions of Eq. (20) for various values of $J$ were first done in 1964 on an IBM 
mainframe ${ }^{8}$. Recent computations by Chen and Arnush ${ }^{6}$ on a personal computer are in agreement with the earlier results, which have been fitted with analytic functions ${ }^{9}$ for easy application. In the limit $\rho \rightarrow \infty$, Eq. (21) has the asymptotic form $\eta \approx J^{2} / \rho^{2}$. Starting with this solution, one can integrate Eq. (21) inwards to obtain the potential profile $\eta(\rho)$. Examples for two values of $J$ are shown in Fig. 9. These are universal curves which apply to all probe radii $R_{\mathrm{p}}$, characterized by the important parameter $\xi_{\mathrm{p}} \equiv R_{\mathrm{p}} / \lambda_{\mathrm{D}}$ [Eq. (15)].

The part of the curve for $\rho<\xi_{\mathrm{p}}$ is irrelevant, and the part for $\rho>\xi_{\mathrm{p}}$ is unchanged by the probe, since all incoming ions are absorbed by the probe, and therefore $n_{\mathrm{i}}$ and $n_{\mathrm{e}}$ are not affected by the presence of a probe. This is not true if the ions have angular momentum and can orbit the probe; in that case the more complicated theory of Bernstein and Rabinowitz $(\mathrm{BR})^{10}$ must be used. For a given value of $\xi_{\mathrm{p}}$, such as the one shown in Fig. 9, the probe potential for given $J$ is the value of $\eta\left(\xi_{\mathrm{p}}\right)$ on the curve for that $J$. Thus, in our example, $\eta \approx 20$ for $J=10$ and $\approx 1200$ for $J=50$. An $I_{i}-V_{p}$ characteristic for given $\xi_{\mathrm{p}}$ is generated by varying $J$ and cross-plotting. Note that $\xi_{\mathrm{p}}$ itself depends on the unknown value of $n_{0}$, and the determination of $n$ from $J$ generally requires iteration. $J \xi_{\mathrm{p}}$, however, is independent of $n_{0}$, and this quantitiy is often plotted instead of $J$. In this cylindrical case, no artificial sheath edge is introduced, and hence there is no confusion between $\lambda_{D}$ and $\lambda_{\text {Ds }}: \lambda_{D}$ is always evaluated using the density $n_{0}$ at infinity.

For the present application, we need to find $I_{\mathrm{i}}\left(V_{\mathrm{f}}\right)$. We start by fixing the value of $J$. The floating condition is $I_{\mathrm{i}}=I_{\mathrm{e}}$, where $I_{\mathrm{e}}$ per unit length is given by

$$
I_{e}=2 \pi R_{p} n_{0} v_{e r} \exp \left(-\eta_{f}\right)
$$

and $I_{\mathrm{i}}$ is given by Eq. (20). Solving for $\eta_{\mathrm{f}}$, we find that $n_{0}$ cancels, and we have

$$
\eta_{f}=\ln \left[\frac{\xi_{p}}{J}\left(\frac{M}{4 \pi m}\right)^{1 / 2}\right]
$$

However, $\eta\left(\xi_{\mathrm{p}}\right)$ must also satisfy the solution $\eta(\rho)$ of Eq. (21) for the given value of $J$ and $\rho$ $=\xi_{\mathrm{p}}$. Fig. 10 shows both $\eta(\rho)$ and $\eta_{\mathrm{f}}\left(\xi_{\mathrm{p}}\right)$ for $J=10$ on the same plot. The intersection gives $\eta_{\mathrm{f}}$ and $\xi_{\mathrm{p}}$ for that value of $J$. By varying $J$, one can generate the functions $\eta_{\mathrm{f}}\left(\xi_{\mathrm{p}}\right)$ and $J\left(\eta_{\mathrm{f}}\right)$ for given $\xi_{\mathrm{p}}$, the dimensionless versions of $V_{\mathrm{f}}\left(R_{\mathrm{p}}\right)$ and $I_{\mathrm{i}}\left(V_{\mathrm{f}}\right)$ for given $R_{\mathrm{p}}$. Also shown in Fig. 10 is the position of the classical sheath edge, as usually defined at $\eta=1 / 2$. Contrary to the plane case, however, the ion density there is not $n_{0} \exp (-1 / 2)$ because $n_{\mathrm{i}} \neq n_{\mathrm{e}}$ at that point. The exact cylindrical solution does not require the arbitrary assumption that quasineutrality holds up to the sheath edge.

We can, however, define an effective collection radius ("sheath" radius) $R_{\mathrm{s}}$, such that if the Bohm criterion were satisfied there, the correct ion current would flow to the probe. The cylindrical equivalent of Eq. (1) is

$$
I_{i}=2 \pi R_{s} \alpha_{0} n_{0} c_{s}
$$

$I_{\mathrm{i}}$ can also be expressed in terms of $J$ by Eq. (20). Equating these two expressions, one can solve for $R_{\mathrm{s}}$, obtaining

$$
\alpha_{0}\left(R_{s} / R_{p}\right)=\sqrt{2} J / \xi_{p} .
$$


If we now define

$$
\alpha \equiv \sqrt{2} J / \xi_{p}=\alpha_{0}\left(R_{S} / R_{p}\right),
$$

Eq. (24) takes its usual form with $\alpha$ replacing $\alpha_{0}$ :

$$
I_{i}=2 \pi R_{p} \alpha n_{0} c_{s} .
$$

Thus, $\alpha$ can be considered a cylindrically modified value of the Bohm coefficient $\alpha_{0}$, and the ratio $\alpha / \alpha_{0}$ is a measure of the expansion of the collection area beyond the probe area when the probe is at the floating potential. The position of $R_{\mathrm{s}}$ is shown in Fig. 10; it is $n o t$ at $\eta=$ $1 / 2$. Computed values of $\eta_{\mathrm{f}}, \alpha$, and $\alpha / \alpha_{0}$ as functions of $\xi_{\mathrm{p}}$ are shown in Fig. 11. Note that $\eta_{\mathrm{f}}$ approaches the plane-geometry value of 5.18 and $\alpha / \alpha_{0} \rightarrow 1$ as $\xi_{\mathrm{p}} \rightarrow \infty$. From Eqs. (20) and (26) we can solve for $n_{0}$, obtaining

$$
n_{0}=N_{i}(A B R)=\frac{I_{i}\left(V_{f}\right)}{2 \pi R_{p} c_{S} \alpha},
$$

which can evaluated from the extrapolated $I_{\mathrm{i}}\left(V_{\mathrm{f}}\right)$ per unit length once $R_{\mathrm{p}}$ and $K T_{\mathrm{e}}$ are known. The value of $\alpha\left(\xi_{\mathrm{p}}\right)$ can be found from the following analytic fit to the curve in Fig. 11:

$$
\alpha \approx 0.607+2432 / \exp \left(7.01 \xi_{p}^{0.096}\right) .
$$

Since $\xi_{\mathrm{p}}$ depends on $n_{0}$, however, solution of Eq. (28) requires iteration.

\section{COMPARISON WITH EXPERIMENT}

The procedure for finding $V_{\mathrm{s}}$ and $K T_{\mathrm{e}}$ from the $I-V$ curve, as well as the density $N_{\mathrm{e}}$ from the electron saturation current, was described following Eq. (2). The density $N_{\mathrm{i}}(\mathrm{CL})$ can then be computed from Eq. (4) using $I_{\mathrm{i}}\left(V_{\mathrm{f}}\right)$. For comparison, the density according to ABR theory can be found from Eq. (28). All of these steps have been automated on an Excel $^{\circledR}$ spreadsheet to give $V_{\mathrm{s}}, K T_{\mathrm{e}}, N_{\mathrm{e}}, N_{\mathrm{i}}(\mathrm{CL})$, and $N_{\mathrm{i}}(\mathrm{ABR})$ from a 1000 -point $I-V$ curve in $<<1 \mathrm{sec}$. Here we present data taken in a 2-MHz argon inductively coupled plasma with an $\mathrm{RF}$-compensated Langmuir probe $.015 \mathrm{~cm}$ in diam and $1 \mathrm{~cm}$ long at various pressures and RF powers. The probe densities are compared with those from microwave interferometry. Details of the experiment are described elsewhere ${ }^{11}$. The data in Fig. 12 span a range of $\xi_{\mathrm{p}}$ from $\approx 1$ to 4.5 . The floating-potential-CL method gives $n$ values agreeing with those from microwaves to within about $20 \%$, while the ABR method gives consistently lower $n$. The electron densities $N_{\mathrm{e}}$ behave sporadically, as one would expect from their sensitivity to the chosen value of $V_{\mathrm{s}}$.

In these analyses, $I_{\mathrm{i}}$ was extrapolated to $V_{\mathrm{f}}$ by fitting a straight line to the $I_{\mathrm{i}}^{\mathrm{p}} v s . V_{\mathrm{p}}$ plot, where $p=4 / 3$. A fit can also be made for other values of $p$. In particular, Langmuir's orbital-motion-limited theory ${ }^{2}$ predicts $p=2$, and solutions ${ }^{8}$ of the ABR equation show $p \geq 2$. Indeed, as shown in the example of Fig. 13, at low densities $I_{\mathrm{i}}{ }^{2}$ fits better to a straight line than does $I_{\mathrm{i}}^{4 / 3}$, suggesting that some ion orbiting is taking place. Nonetheless, fitting with $p=$ $4 / 3$ gives more reasonable values of $n$. In this example, $p=4 / 3$ yields $n_{11}=0.734$ while $p=$ 2 gives $n_{11}=0.475$, compared with a microwave density of 0.872 , where $n_{11}$ is in units of $10^{11} \mathrm{~cm}^{-3}$. The sensitivity of $N_{\mathrm{i}}(\mathrm{CL})$ and $N_{\mathrm{i}}(\mathrm{ABR})$ to $p$ is shown in Fig. 14 for the 2 mTorr, 
$900 \mathrm{~W}$ point of Fig. 12. We see that $p=4 / 3$ gives yields better agreement between $N_{\mathrm{i}}(\mathrm{CL})$ and the microwave measurement than does $p=2$ or $1 / 2$. This is generally true of the cases we have examined, though $p=4 / 3$ has no theoretical justification.

\section{DISCUSSION}

The FP-CL method for cylindrical probes neglects three major effects: 1) electron density and cylindrical curvature in the calculation of sheath thickness; 2) orbiting of ions around the probe; and 3) loss of ions moving in the $z$ direction, parallel to the probe axis. We have treated (1) with the ABR analysis, finding that it yields values of $n$ that are too low. The reason is probably that some ions have enough angular momentum to orbit the probe and miss it. Taking full account of this effect with the BR theory ${ }^{10}$, however, yields $n$ values that are too high. We have previously suggested ${ }^{1}$ that a few collisions in the presheath can greatly decrease the amount of orbiting, and that the geometric mean between $n(\mathrm{ABR})$ and $n(\mathrm{BR})$ gives a good approximation in this case of partial orbiting. The FP-CL method apparently succeeds because of a fortuitous cancellation of effects (1) and (2).

This one-dimensional treatment fails to account for the finite length of the probe. When $\xi_{\mathrm{p}}$ is small, incoming ions are crowded together and form a large positive space charge near the probe surface. As shown in Fig. 15, this space charge creates an electric field $E_{\mathrm{z}}$, driving the ions out past the end of the probe. The $n_{\mathrm{i}}(r)$ and $n_{\mathrm{e}}(r)$ profiles calculated with the ABR theory for $J=10$ are shown in Fig. 16. At the radius of a floating probe, there is an appreciable excess ion charge. For large $V_{\mathrm{p}}$ this value of $J$ corresponds to a very small probe, and the ion charge can be extremely large unless the ions can escape axially. Also shown in Fig. 16 are the radius where $\eta=1 / 2$ and the effective sheath radius $R_{\mathrm{s}}$. It is clear that quasineutrality does not hold down to $\eta=1 / 2$ in the cylindrical case.

We can make a rough estimate of the endloss as follows. Let $\langle\eta\rangle$ be an average potential in the sheath. The radial ion velocity is given by Eq. (17):

$$
v_{r}=c_{S} \sqrt{2<\eta>} \text {. }
$$

With $R_{\mathrm{s}}$ defined as in Eq. (24), ion continuity gives, for $r \approx R_{\mathrm{p}}$,

$$
n_{i} v_{r} R_{p}=\alpha_{0} n_{0} c_{s} R_{s}
$$

Using Eq. (26), we have for the average ion density in the sheath

$$
<n_{i}>\approx \frac{\alpha_{0} n_{0}}{(2<\eta>)^{1 / 2}} \frac{R_{s}}{R_{p}}=\frac{\alpha n_{0}}{(2<\eta>)^{1 / 2}} .
$$

Since $\eta$ now varies along $z$, its $z$-average is perhaps $1 / 2<\eta>$, so that the ions escaping axially will have an average velocity

$$
<v_{z}>\approx c_{S}<\eta>^{1 / 2}
$$

Thus, the loss flux to both ends is given by 


$$
I_{\text {loss }}=2 \pi\left(R_{S}^{2}-R_{p}^{2}\right)<n_{i}><v_{z}>\sqrt{2} \pi\left(R_{S}^{2}-R_{p}^{2}\right) \alpha n_{0} c_{s}
$$

Note that $\langle\eta\rangle$ has canceled out, so that it does not have to be evaluated precisely. The total radial flux from ABR theory is given by Eq. (27):

$$
I_{i}=2 \pi R_{p} L \alpha n_{0} c_{s}
$$

where $I_{\mathrm{i}}$ now includes the probe length. With the help of Eq. (26), we can now write

$$
\frac{I_{\text {loss }}}{I_{i}}=\frac{\sqrt{2} \pi\left(R_{s}^{2}-R_{p}^{2}\right) \alpha n_{0} c_{s}}{2 \pi R_{p} L \alpha n_{0} c_{s}}=\frac{1}{\sqrt{2}} \frac{R_{p}}{L}\left(\frac{R_{s}^{2}}{R_{p}^{2}}-1\right)=\frac{1}{\sqrt{2}} \frac{R_{p}}{L}\left(\frac{\alpha^{2}}{\alpha_{0}^{2}}-1\right) .
$$

From Fig. 11, we see that for $\xi_{\mathrm{p}}=1$ (the worst case), $\left(\alpha / \alpha_{0}\right)^{2}$ is of order 20 . For the probe dimensions used here, $R_{\mathrm{p}} / L=.0075$. The endloss correction of Eq. (36) then amounts to $\approx 11 \%$. However, it could be larger with thicker probes.

In conclusion, we have found a rapid method for analyzing probe characteristics which gives more accurate ion density measurements in RF plasmas than any existing probe theory. The method is entirely heuristic and is not supported by a detailed treatment of the sheath. It apparently works because of the self-cancellation of neglected effects. 


\section{FIGURE CAPTIONS}

Fig. 1. Typical $I-V$ characteric taken with a probe $0.15 \mathrm{~mm}$ in diameter and $1 \mathrm{~cm}$ long.

Fig. 2. First derivative of the $I-V$ curve. The points have been smoothed over the digitization noise, and the line is a further smoothing of the data.

Fig. 3. Example of a case where the "knee" of the $I-V$ curve is ill-defined.

Fig. 4. Ion current $I_{\mathrm{i}}^{4 / 3}$ vs. $V$, and a least-squares fitted straight line. The electron current has not been subtracted from the $I_{\mathrm{i}}$ data. The intersection of the line with the vertical line at floating potential $V_{\mathrm{f}}$ yields the value of $I_{\mathrm{i}}\left(V_{\mathrm{f}}\right)$ used in the analysis.

Fig. 5. Semi-logarithmic plot of electron current $I_{\mathrm{e}}$ vs. $V$ and the least-squares fitted line. The solid points are the raw data without correction for the ion contribution.

Fig. 6. Calculated potential in a plane sheath with different assumed boundary conditions at the sheath edge $\xi=0$, where $V \equiv 0$.

Fig. 7. The two curves of Fig. 6 on a log-log scale, compared with the normalized ChildLangmuir sheath thickness. The dashed line is the floating potential of a plane probe.

Fig. 8. Normalized sheath radii for various values of $R_{\mathrm{p}} / \lambda_{\mathrm{D}}$ as calculated (points) and as estimated with the CL formula (lines). The right-hand curve of Fig. 7 was used. The dashed line is the floating potential.

Fig. 9. Potential profiles in a cylindrical sheath for two values of normalized probe current $J$. The dashed line is the $J=50$ curve reduced by 10 . The line $\xi_{\mathrm{p}}$ marks the probe radius.

Fig 10. The functions $\eta(\xi)$ and $\eta_{\mathrm{f}}\left(\xi_{\mathrm{p}}\right)$ for $J=10$ in argon. The "classical sheath edge" is the radius at $\eta=0.5$, where the ions have velocity $c_{\mathrm{s}}$. The "effective sheath radius" is that at which the assumed probe current would be collected if the Bohm criterion were satisfied there (which it is not).

Fig. 11. Computed values of $\eta_{\mathrm{f}}=-\mathrm{e} V_{\mathrm{f}} / K T_{\mathrm{e}}, \alpha$, and $\alpha / \alpha_{0}$ for a cylindrical probe in argon and purely radial ion orbits. The curve through the $\alpha$ points is the analytic fit of Eq. (29).

Fig. 12. Probe densities computed with the CL formula $(\square)$, the ABR theory $(\diamond)$, and electron saturation current $(\Delta)$, compared with microwave densities (---) at various pressures.

Fig. 13. Ion current at $1 \mathrm{mTorr}, 450 \mathrm{~W}$ plotted as $I_{\mathrm{i}}^{\mathrm{p}} v s . V_{\mathrm{p}}$ with $p=4 / 3$ (upper curve) and $p=$ 2 lower curve. The straight-line fits are also shown.

Fig. 14. Dependence of calculated density on the exponet $\mathrm{p}$ in $I_{\mathrm{i}}^{\mathrm{p}}-V_{\mathrm{p}}$. Values of $n$ computed with the CL formula and the ABR theory are compared with that measured with microwaves (MW). The star marks the value $p=4 / 3$.

Fig. 15. Schematic of ion space charge around probe tip, causing ions to stream axially.

Fig. 16. ABR solution for the ion ( - ) and electron $(----)$ densities in the sheath for the case $J=10$. The radius of a floating probe is shown by the line labeled $R_{\mathrm{p}}$. Similarly, $R_{1 / 2}$ is the radius where $\eta=1 / 2$, and $R_{\mathrm{S}}$ is the effective sheath radius for this case. For probes at higher $\eta_{\mathrm{p}}$, the line $R_{\mathrm{p}}$ will move to the left for fixed $J$. 


\section{REFERENCES}

${ }^{1}$ F.F. Chen, Phys. Plasmas 8, 3029 (2001).

${ }^{2}$ F.F. Chen, Electric Probes, in "Plasma Diagnostic Techniques", ed. by R.H. Huddlestone and S.L. Leonard (Academic Press, New York), Chap. 4, pp. 113-200 (1965).

${ }^{3}$ M.A. Lieberman and A.J. Lichtenberg, Principles of Plasma Discharges and Materials Processing (Wiley, New York, 1994), p. 165.

${ }^{4}$ J. Gierling and K.-U. Riemann, J. Appl. Phys. 83, 3521 (1998).

${ }^{5}$ I.H. Hutchinson, Principles of Plasma Diagnostics (Cambridge Univ. Press, 1987), p. 61.

${ }^{6}$ F.F. Chen and D. Arnush, Phys. Plasmas 8, 0000 (2001).

${ }^{7}$ J.E. Allen, R.L.F. Boyd, and P. Reynolds, Proc. Phys. Soc. (London) B70, 297 (1957).

${ }^{8}$ F.F. Chen, J. Nucl. Energy, Pt. C 7, 47 (1965).

${ }^{9}$ F.F. Chen, Phys. Plasmas 8, 3029 (2001).

${ }^{10}$ I.B. Bernstein, and I.N. Rabinowitz, Phys. Fluids 2, 112 (1959).

${ }^{11}$ J.D. Evans, W. Zawalsky, and F.F. Chen (in preparation). 


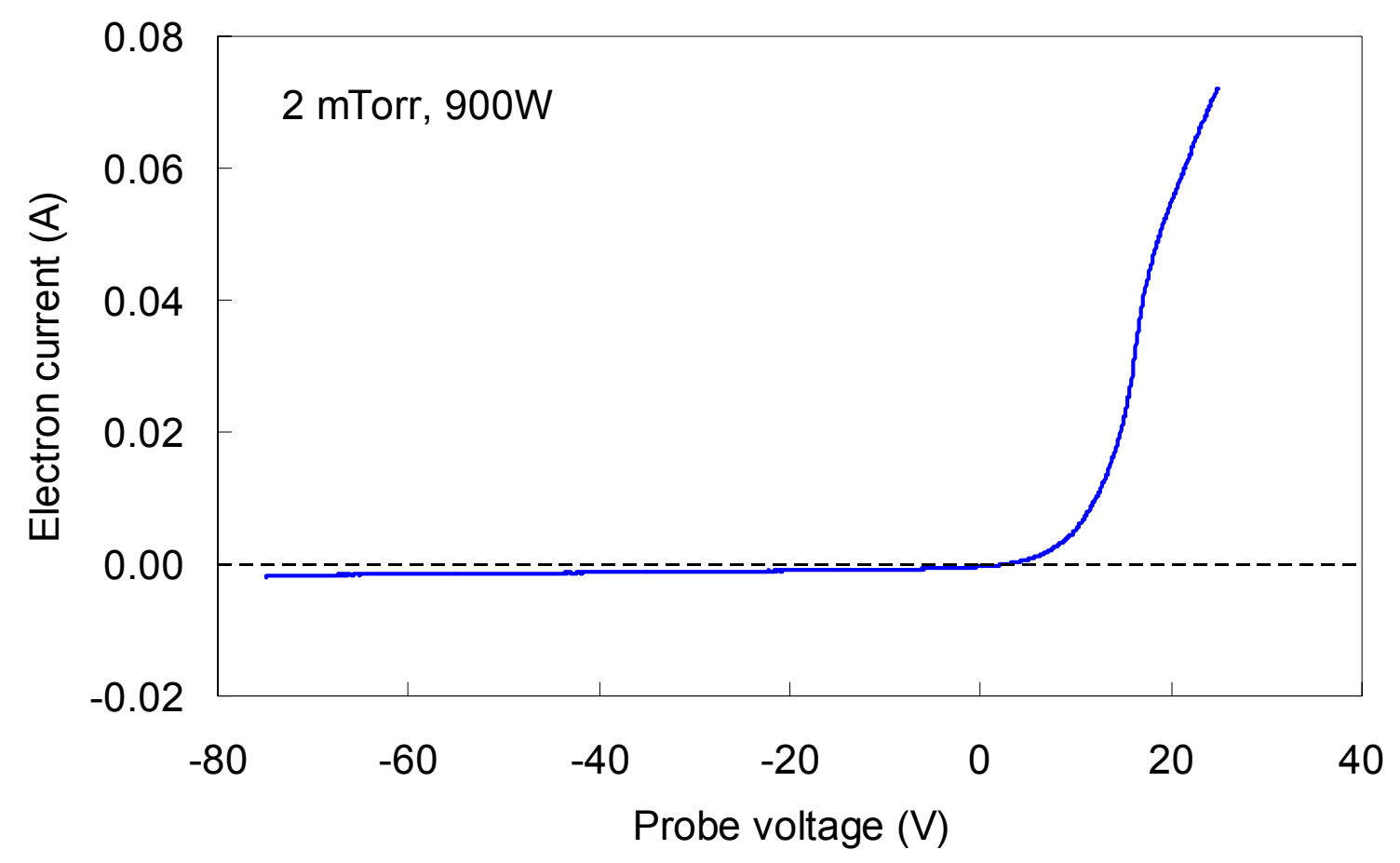

Fig. 1. Typical $I-V$ characteristic taken with a probe $0.15 \mathrm{~mm}$ in diameter and $1 \mathrm{~cm}$ long.

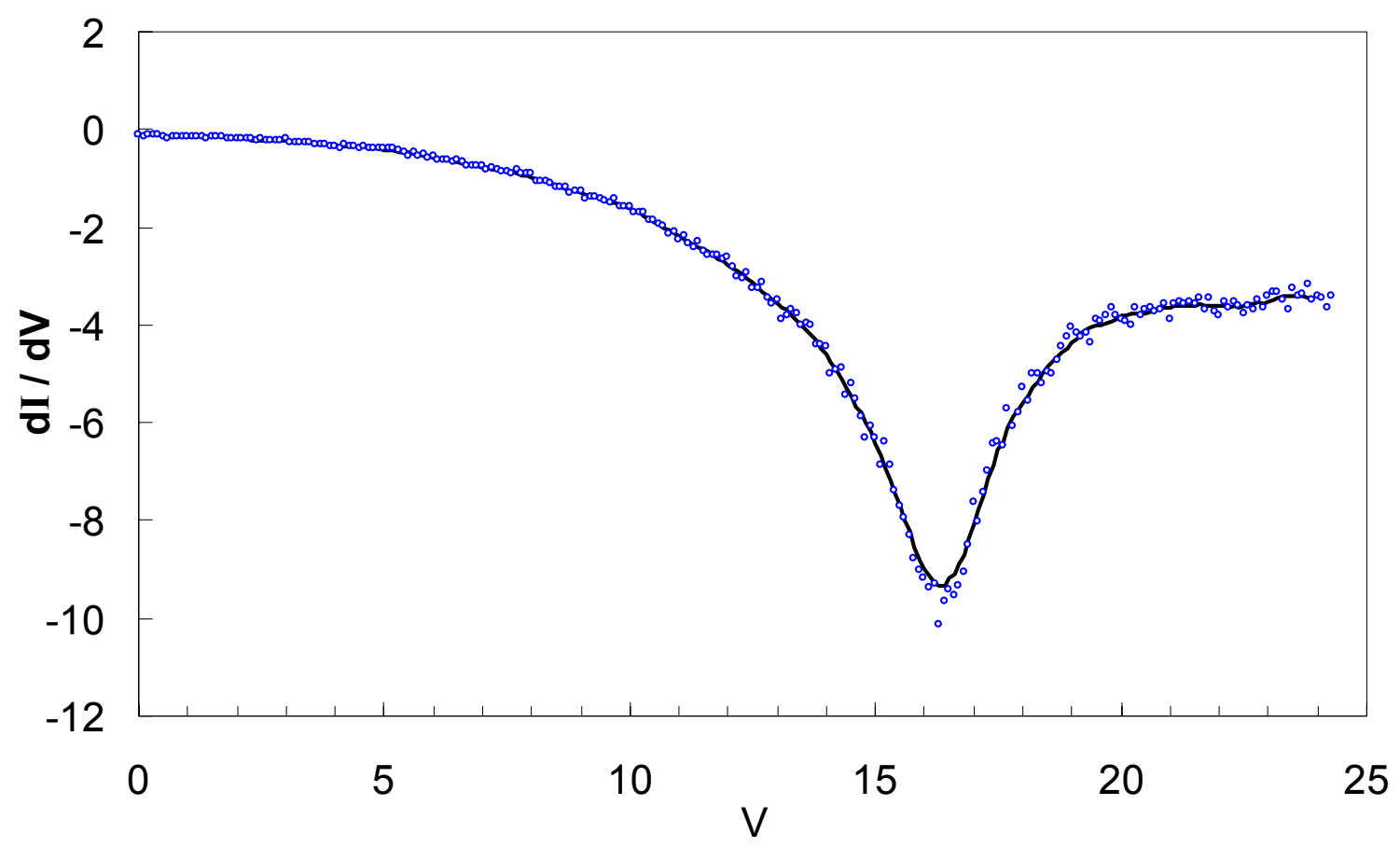

Fig. 2. First derivative of the $I-V$ curve. The points have been smoothed over the digitization noise, and the line is a further smoothing of the data. 


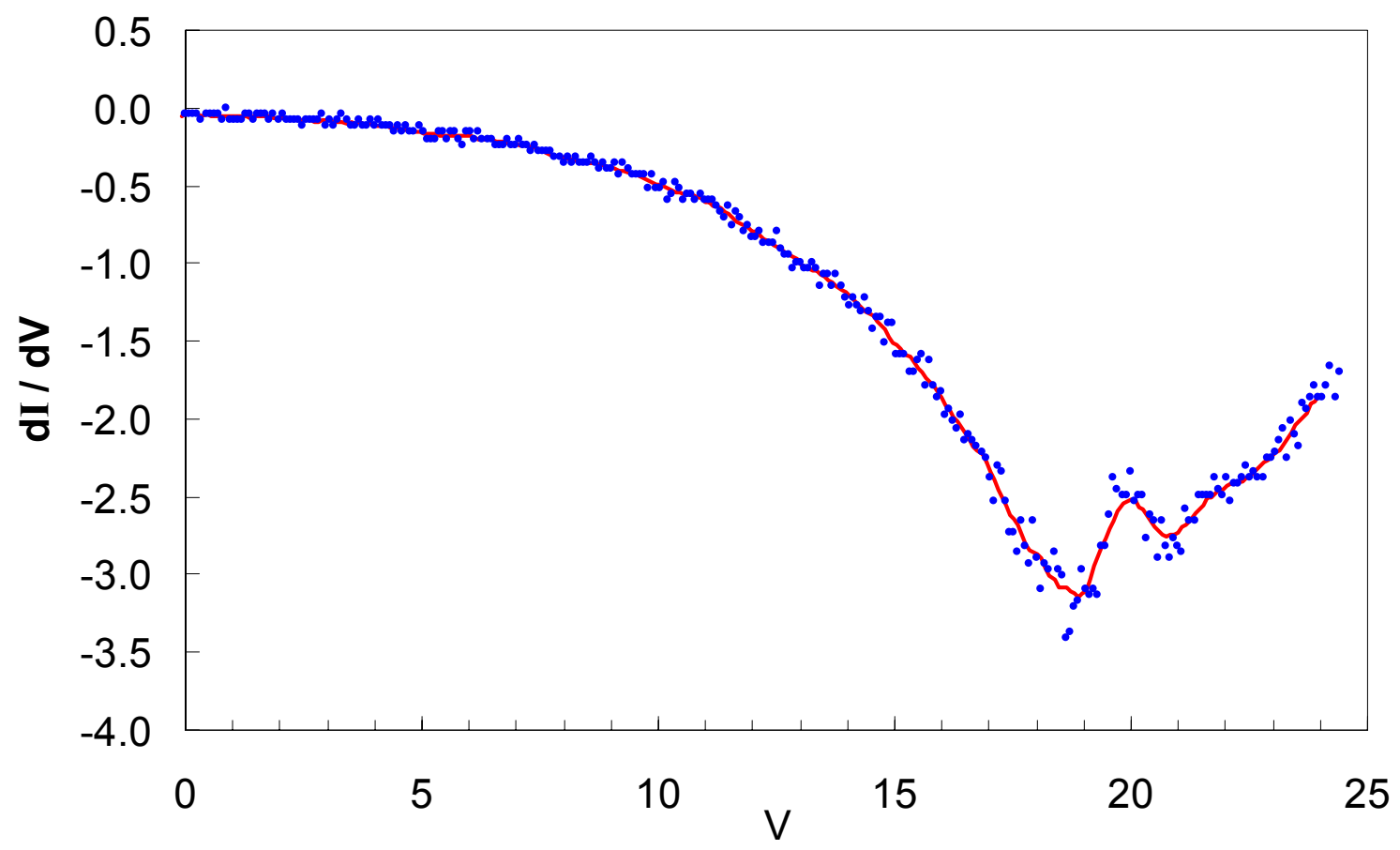

Fig. 3. Example of a case where the "knee" of the $I-V$ curve is ill-defined.

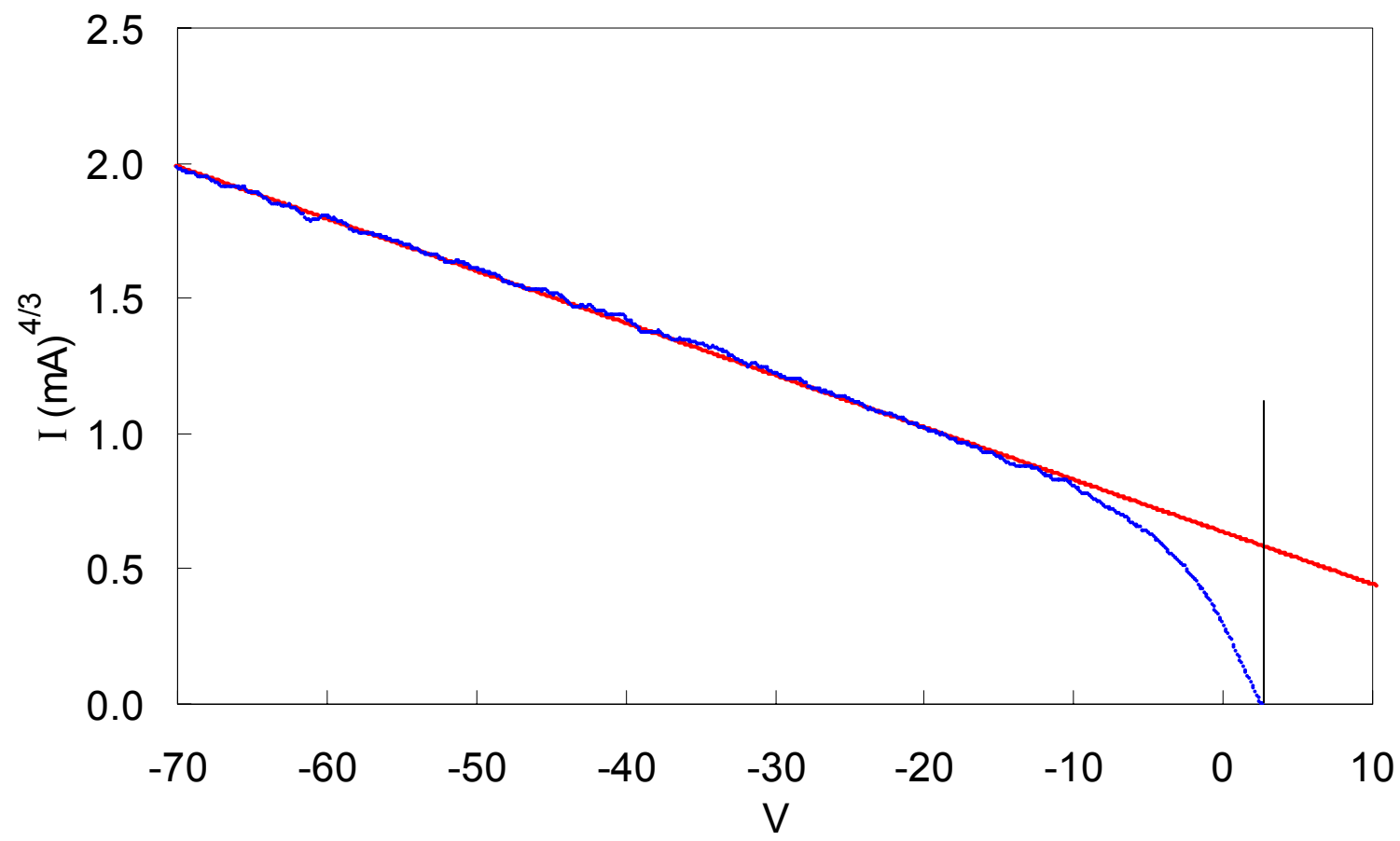

Fig. 4. Ion current $I_{\mathrm{i}}^{4 / 3}$ vs. $V$, and a least-squares fitted straight line. The electron current has not been subtracted from the $I_{\mathrm{i}}$ data. The intersection of the line with the vertical line at floating potential $V_{\mathrm{f}}$ yields the value of $I_{\mathrm{i}}\left(V_{\mathrm{f}}\right)$ used in the analysis. 


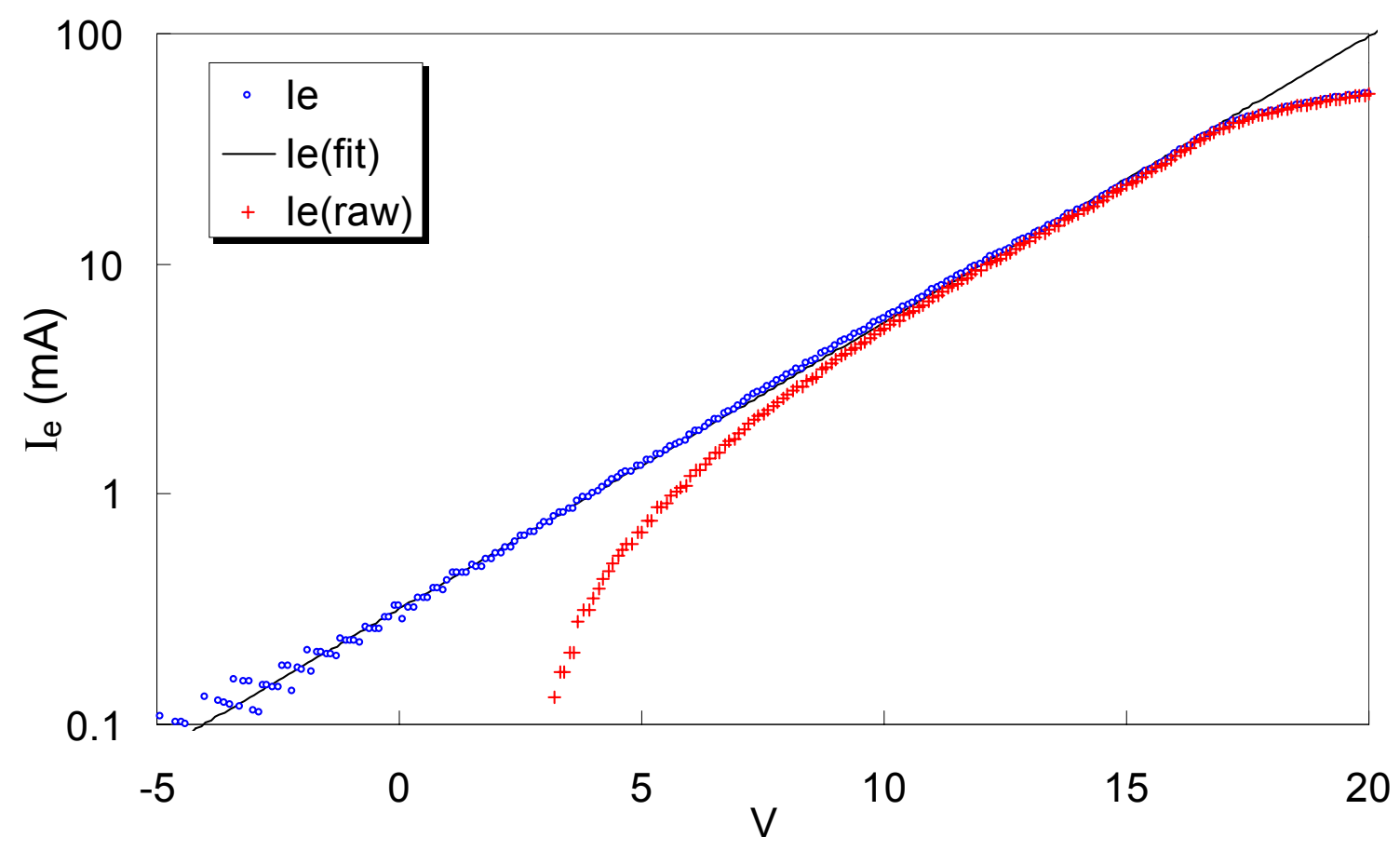

Fig. 5. Semi-logarithmic plot of electron current $I_{\mathrm{e}}$ vs. $V$ and the least-squares fitted line. The solid points are the raw data without correction for the ion contribution.

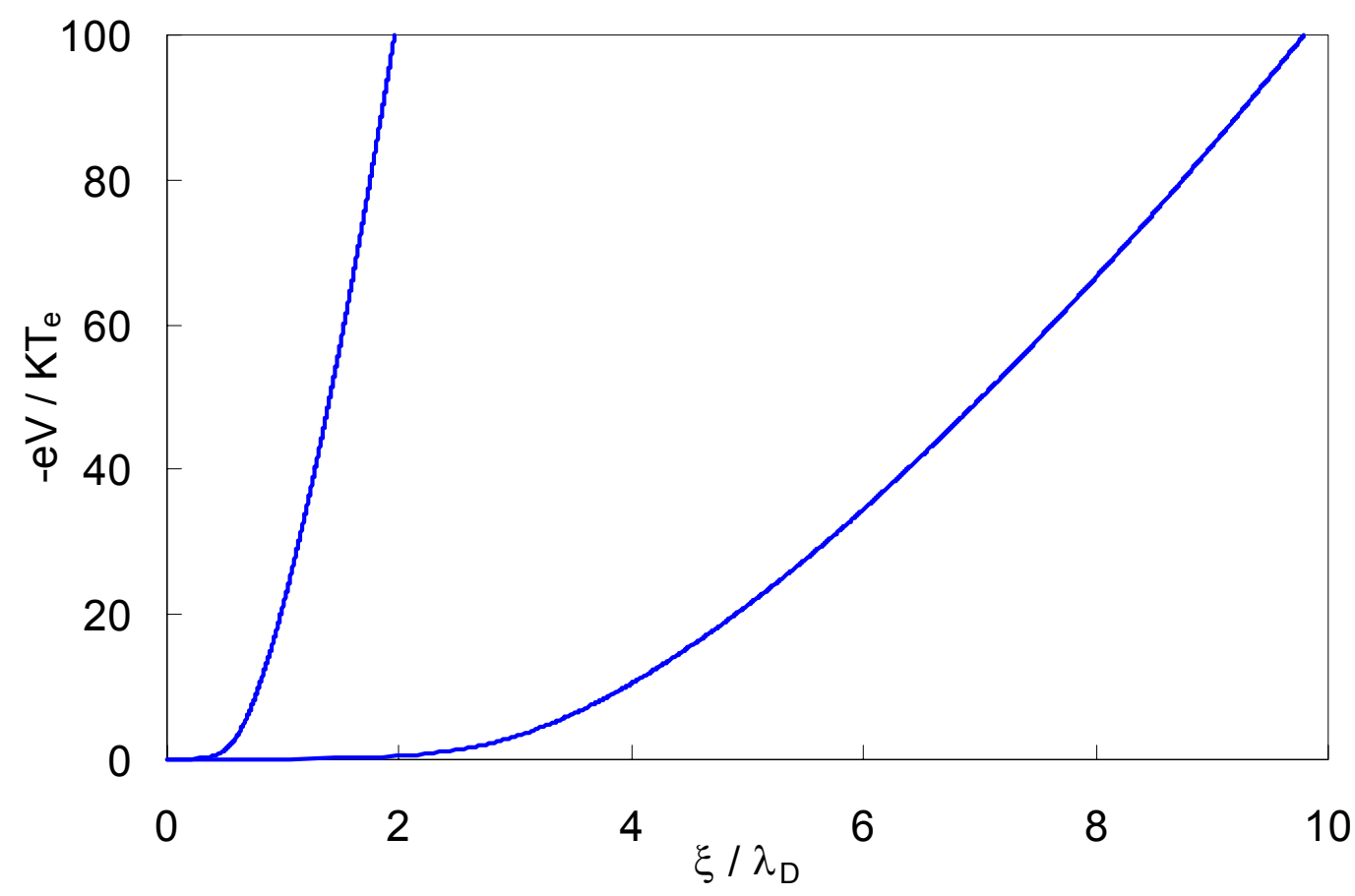

Fig. 6. Calculated potential in a plane sheath with different assumed boundary conditions at the sheath edge $\xi=0$, where $V \equiv 0$. 


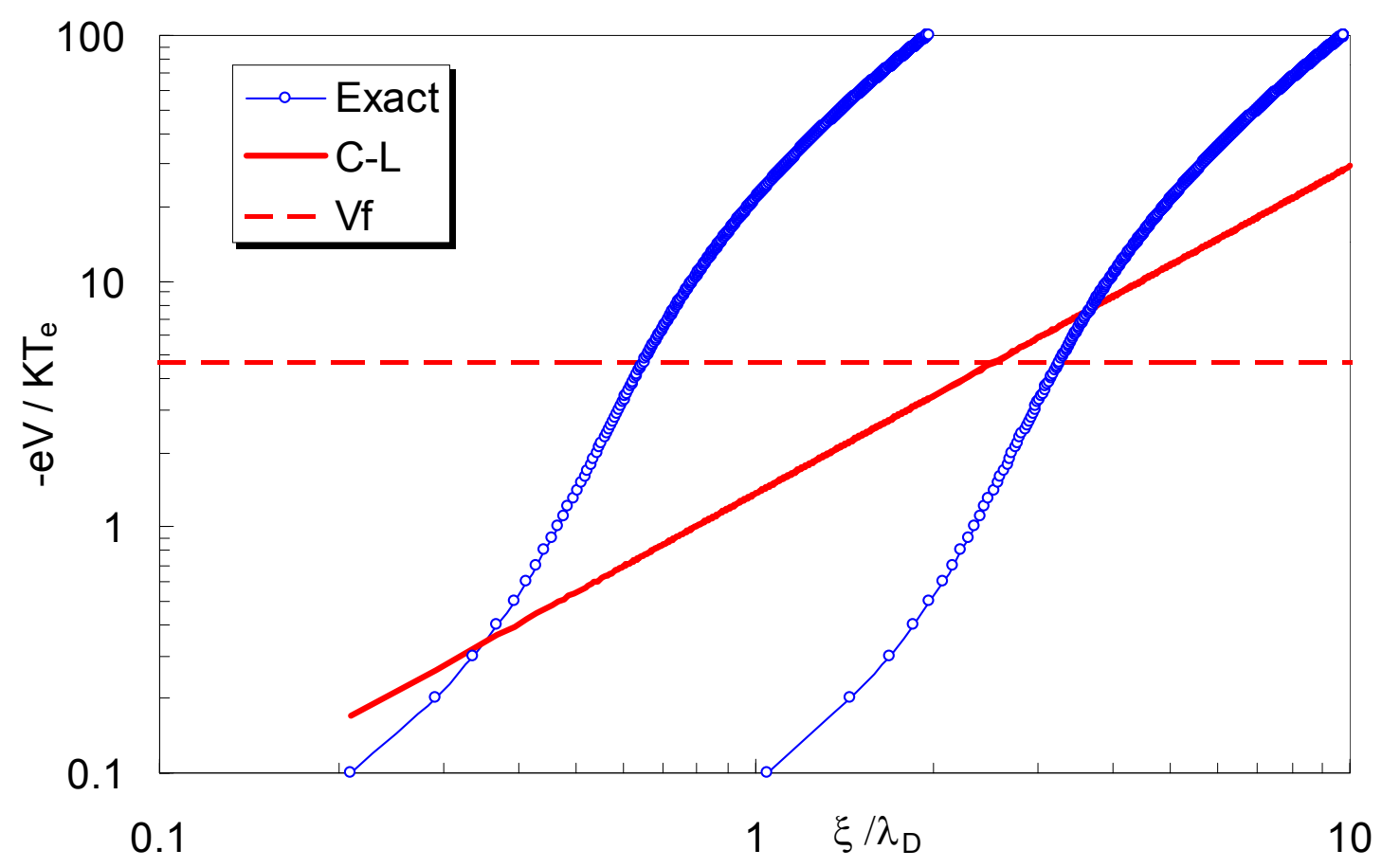

Fig. 7. The two curves of Fig. 6 on a log-log scale, compared with the normalized ChildLangmuir sheath thickness. The dashed line is the floating potential of a plane probe.

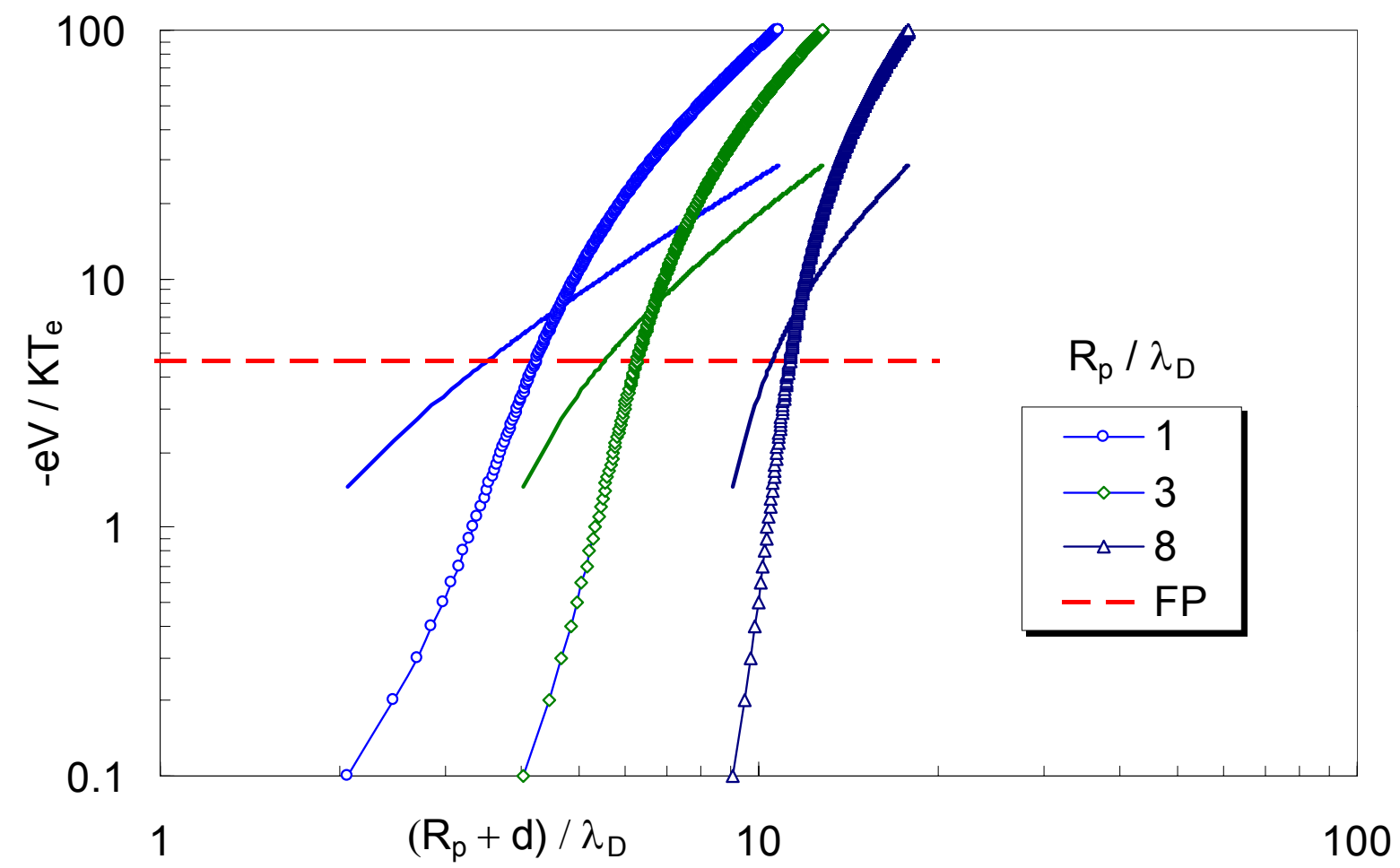

Fig. 8. Normalized sheath radii for various values of $R_{\mathrm{p}} / \lambda_{\mathrm{D}}$ as calculated (points) and as estimated with the CL formula (lines). The right-hand curve of Fig. 7 was used. The dashed line is the floating potential. 


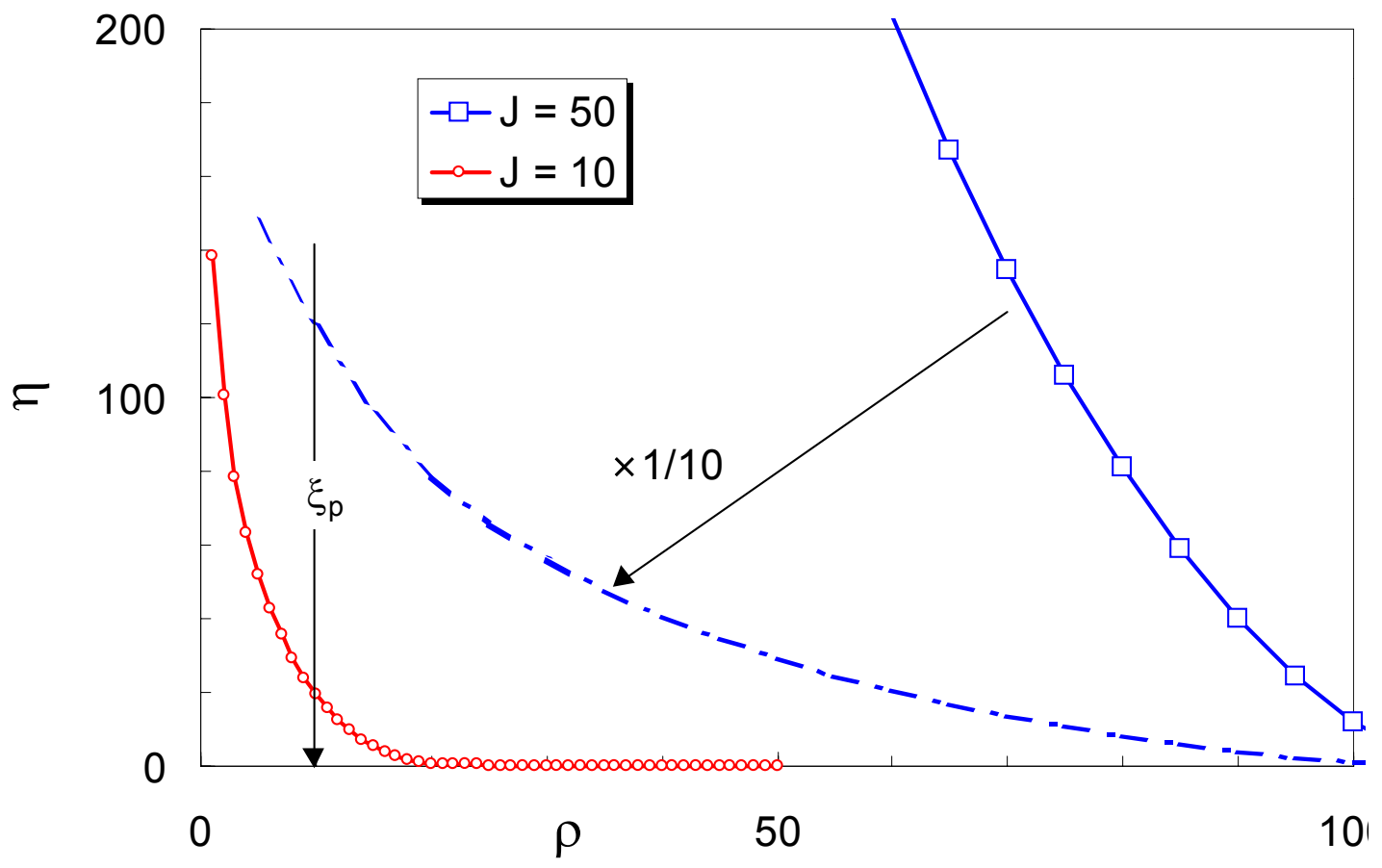

Fig. 9. Potential profiles in a cylindrical sheath for two values of normalized probe current $J$. The dashed line is the $J=50$ curve reduced by 10 . The line $\xi_{\mathrm{p}}$ marks the probe radius.

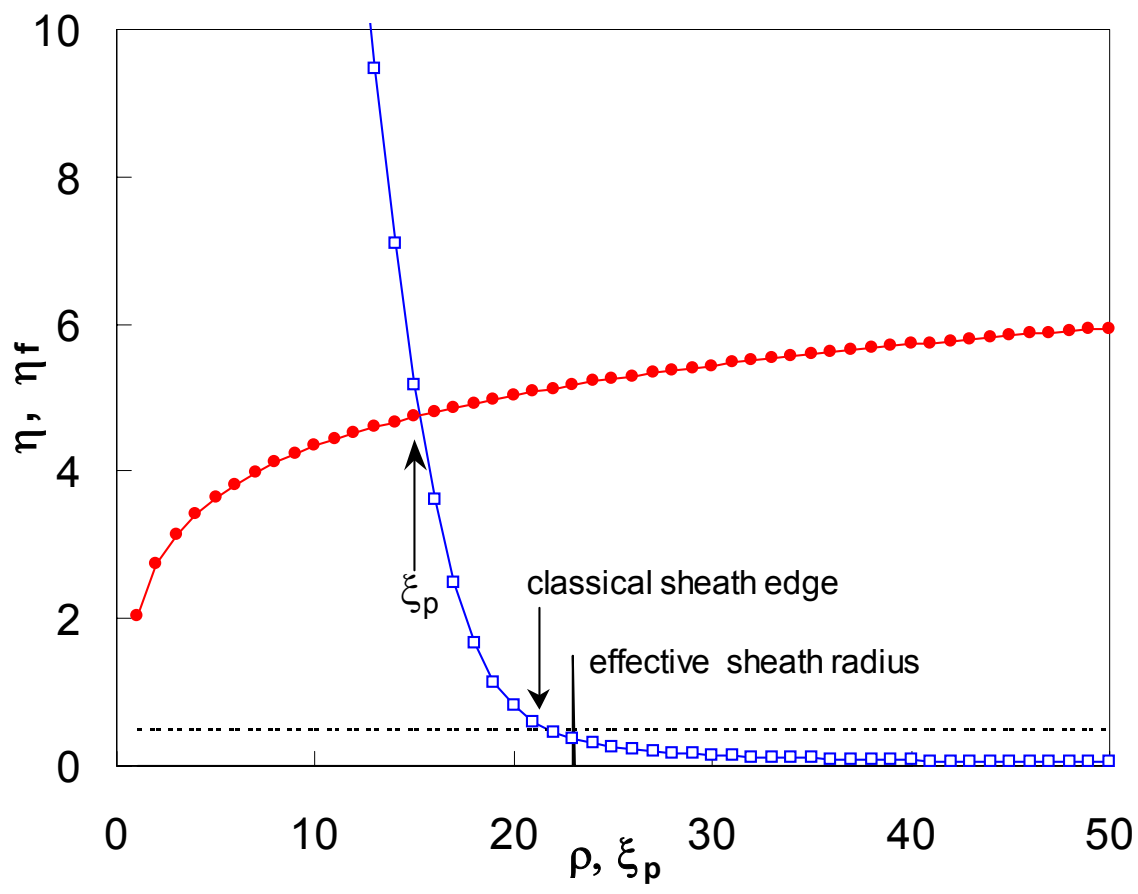

Fig 10. The functions $\eta(\xi)$ and $\eta_{\mathrm{f}}\left(\xi_{\mathrm{p}}\right)$ for $J=10$ in argon. The "classical sheath edge" is the radius at $\eta=0.5$, where the ions have velocity $c_{\mathrm{s}}$. The "effective sheath radius" is that at which the assumed probe current would be collected if the Bohm criterion were satisfied there (which it is not). 


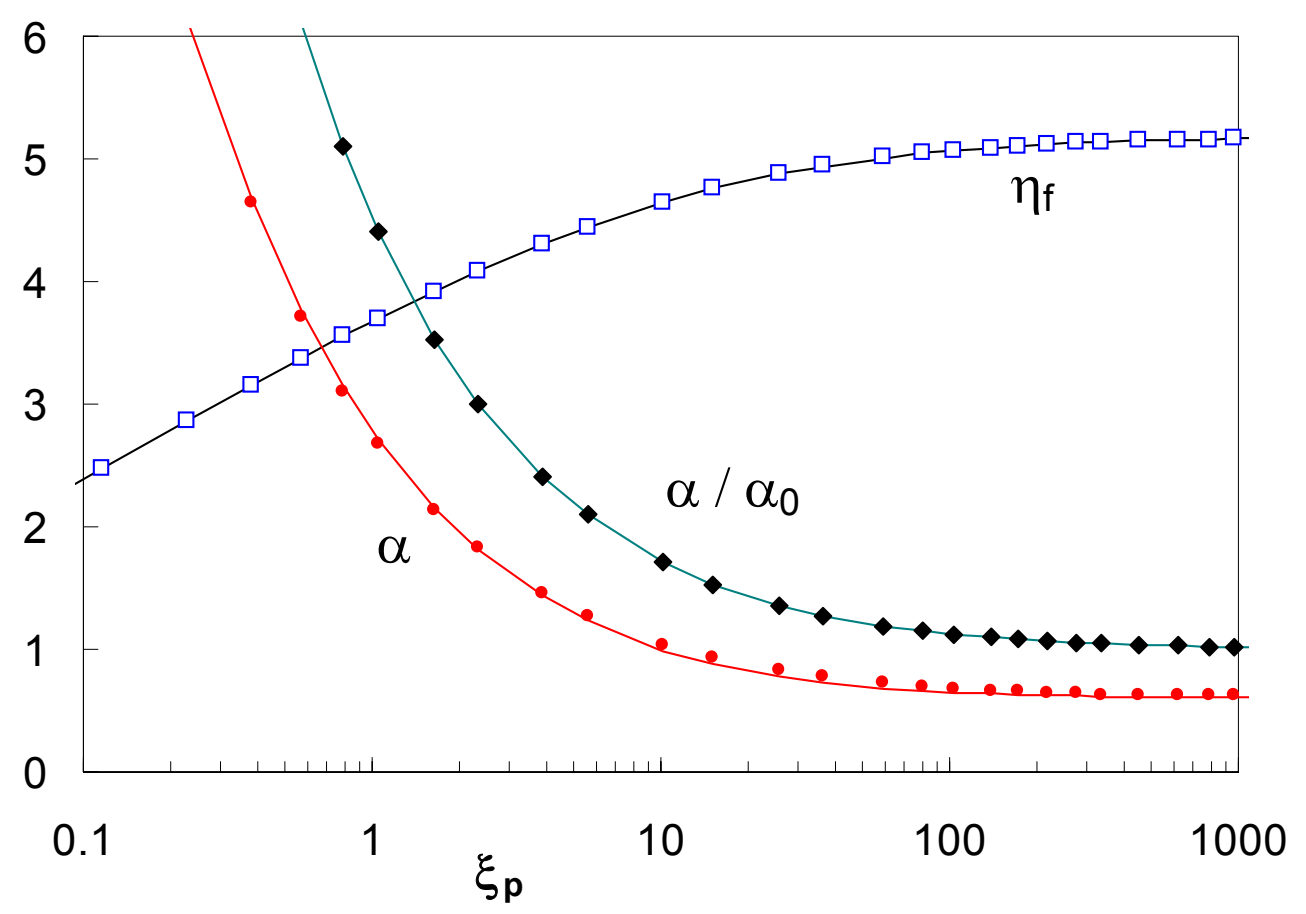

Fig. 11. Computed values of $\eta_{\mathrm{f}}=-\mathrm{e} V_{\mathrm{f}} / K T_{\mathrm{e}}, \alpha$, and $\alpha / \alpha_{0}$ for a cylindrical probe in argon and purely radial ion orbits. The curve through the $\alpha$ points is the analytic fit of Eq. (29). 


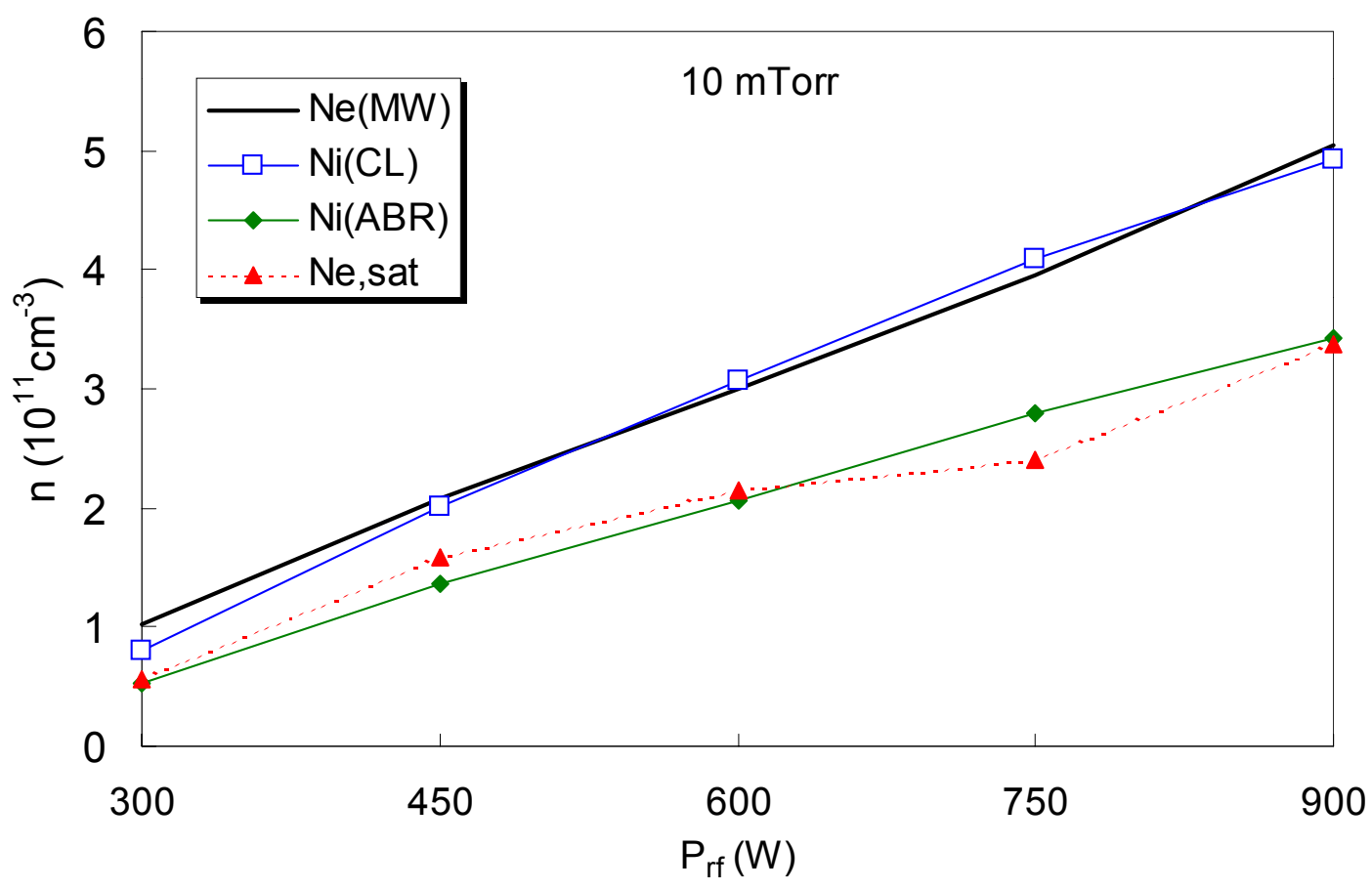

(A)

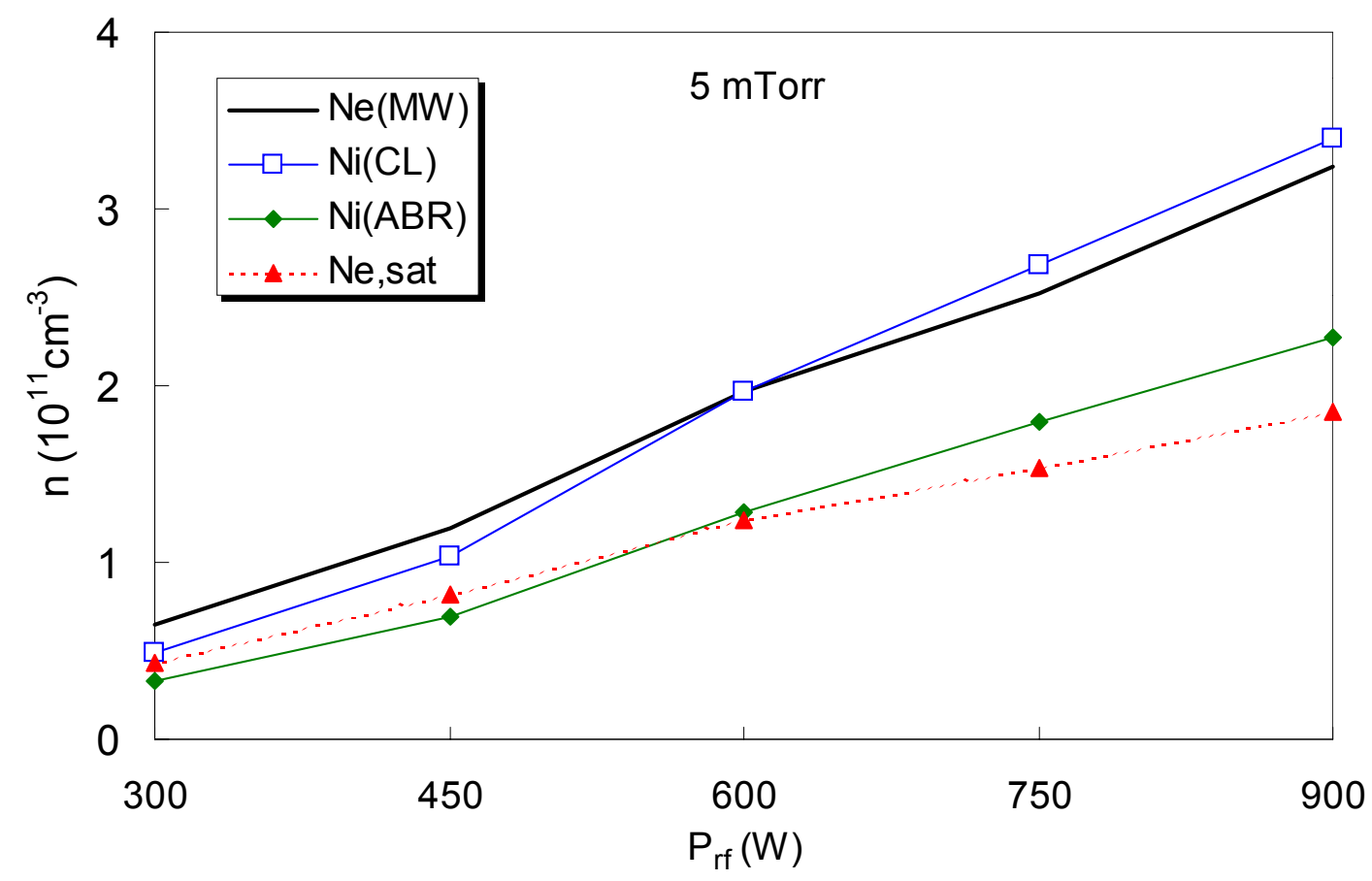

(B) 


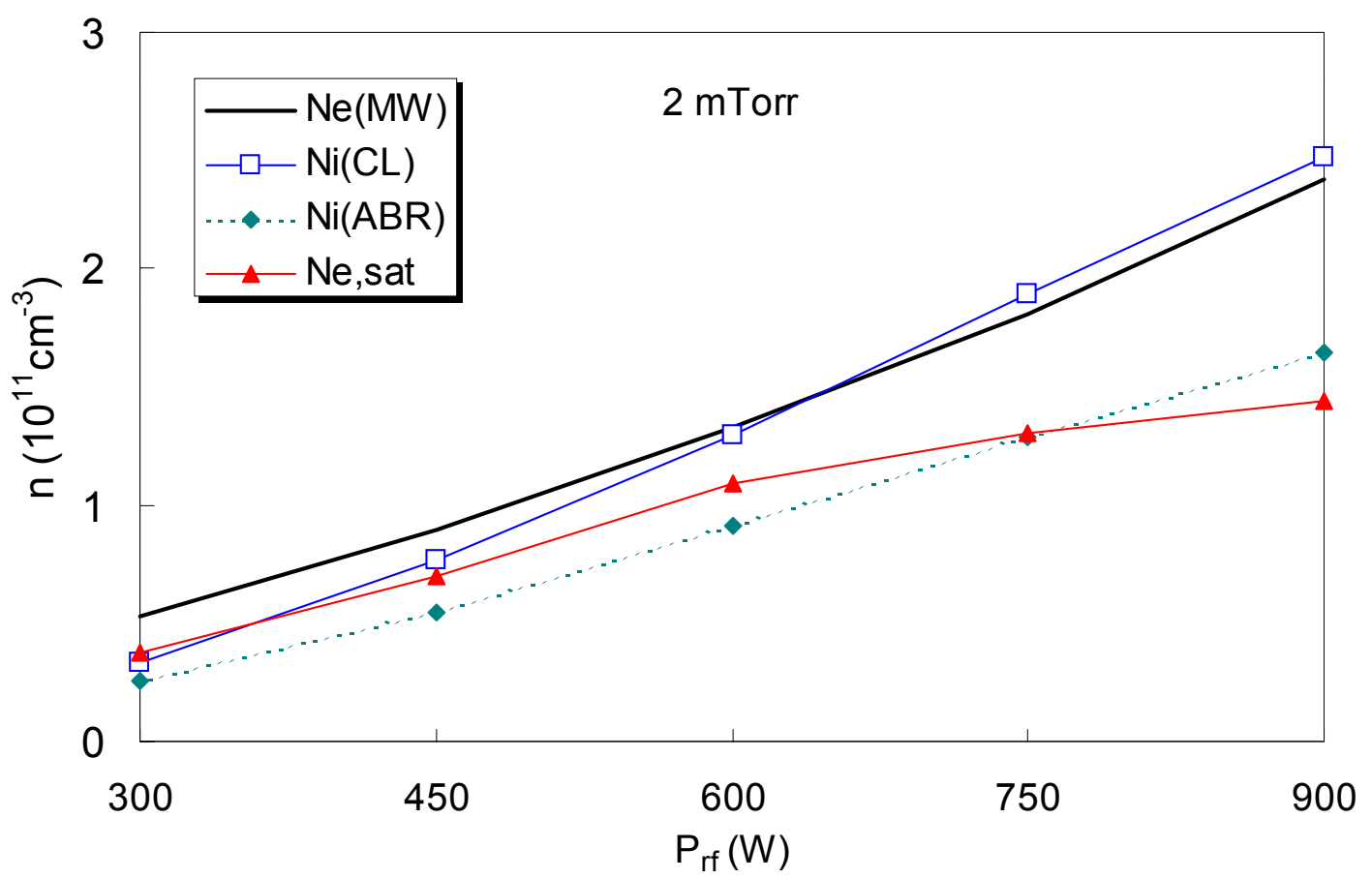

(C)

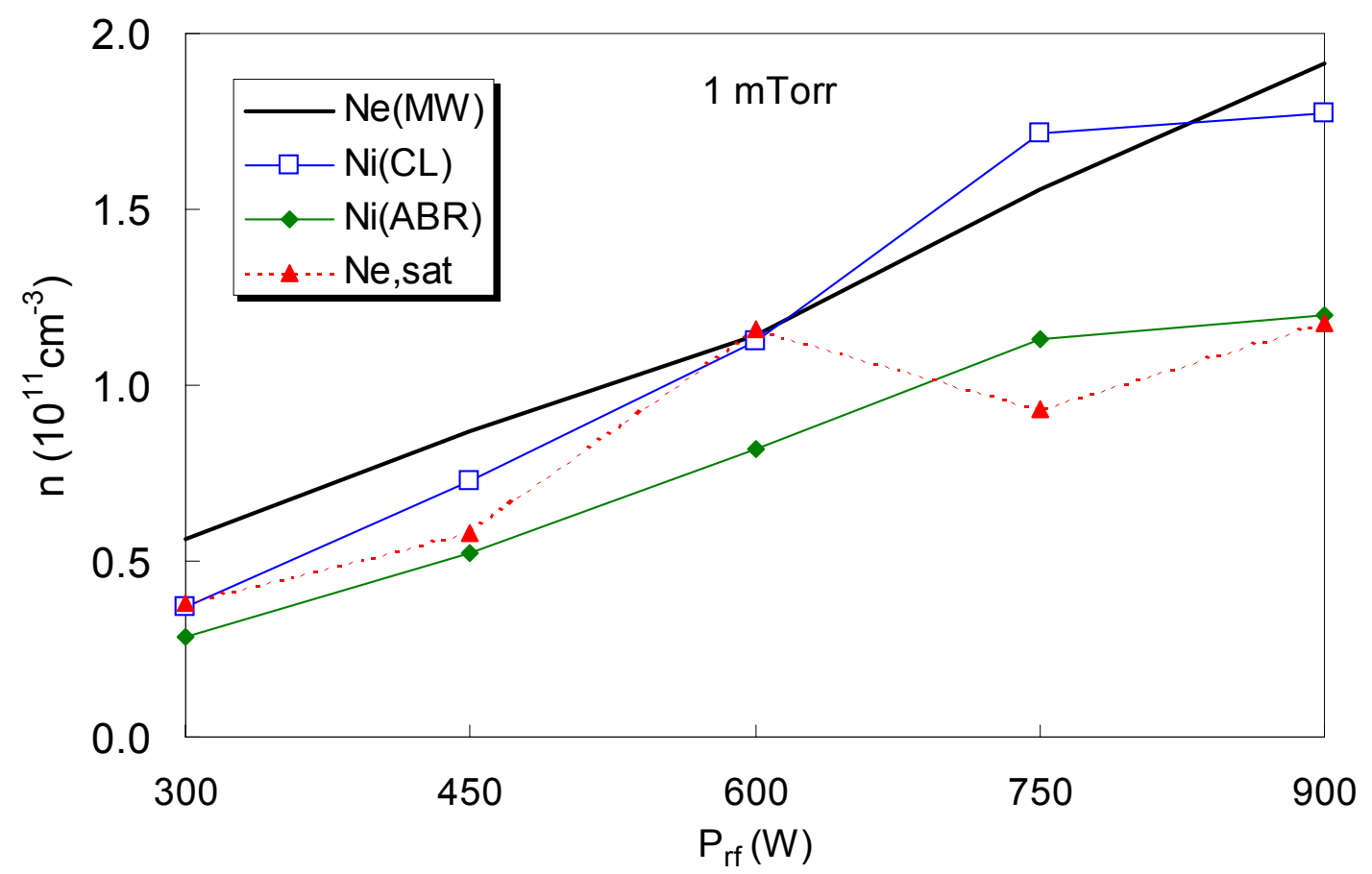

(D)

Fig. 12. Probe densities computed with the CL formula ( $\square$ ), the ABR theory ( $\bullet$ ), and electron saturation current $(\Delta)$, compared with microwave densities (---) at various pressures. 


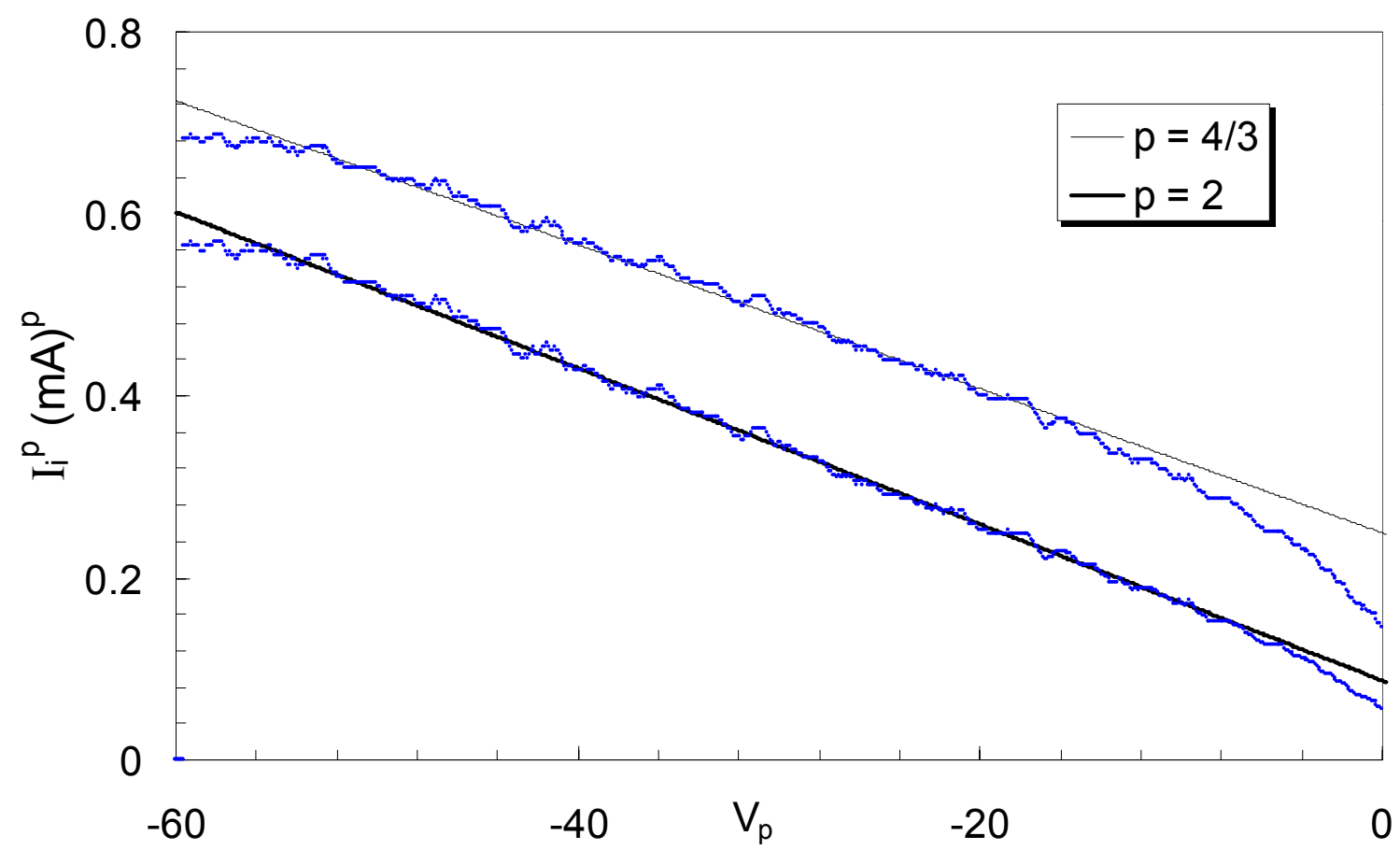

Fig. 13. Ion current at 1 mTorr, $450 \mathrm{~W}$ plotted as $I_{\mathrm{i}}^{\mathrm{p}} v s . V_{\mathrm{p}}$ with $p=4 / 3$ (upper curve) and $p=$ 2 lower curve. The straight-line fits are also shown.

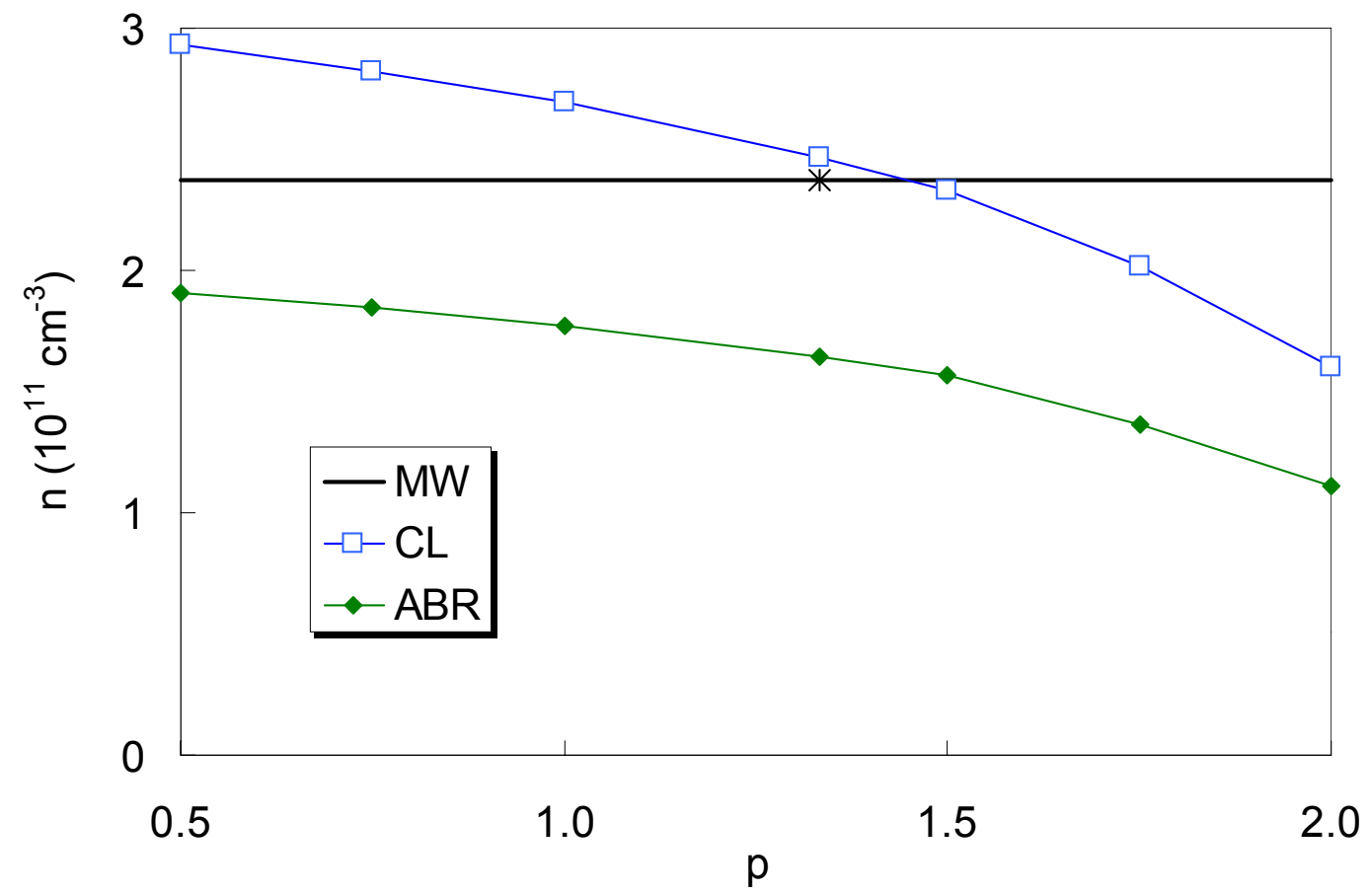

Fig. 14. Dependence of calculated density on the exponent $p$ in $I_{\mathrm{i}}^{\mathrm{p}} \propto V_{\mathrm{p}}$. Values of $n$ computed with the CL formula and the ABR theory are compared with that measured with microwaves (MW). The star marks the value $p=4 / 3$. 


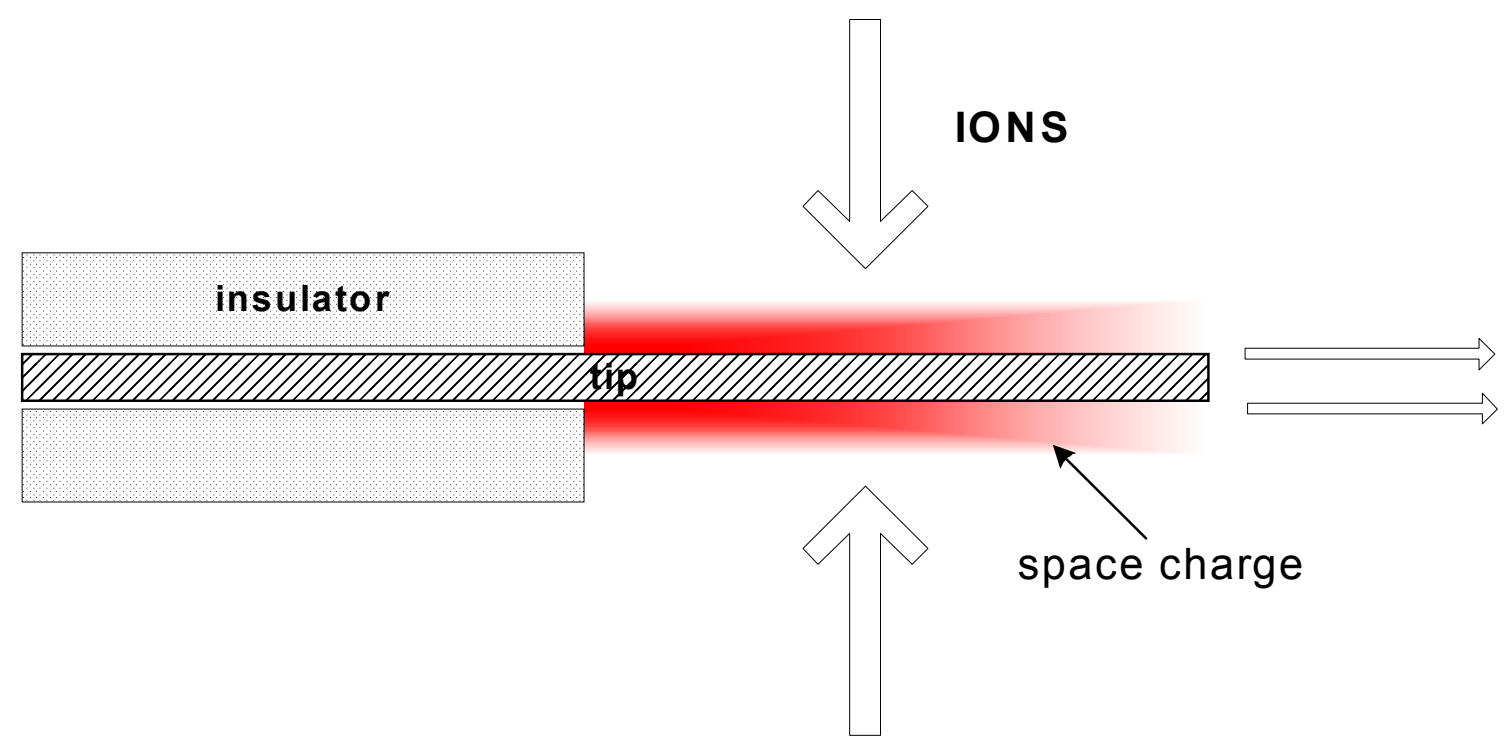

Fig. 15. Schematic of ion space charge around probe tip, causing ions to stream axially.

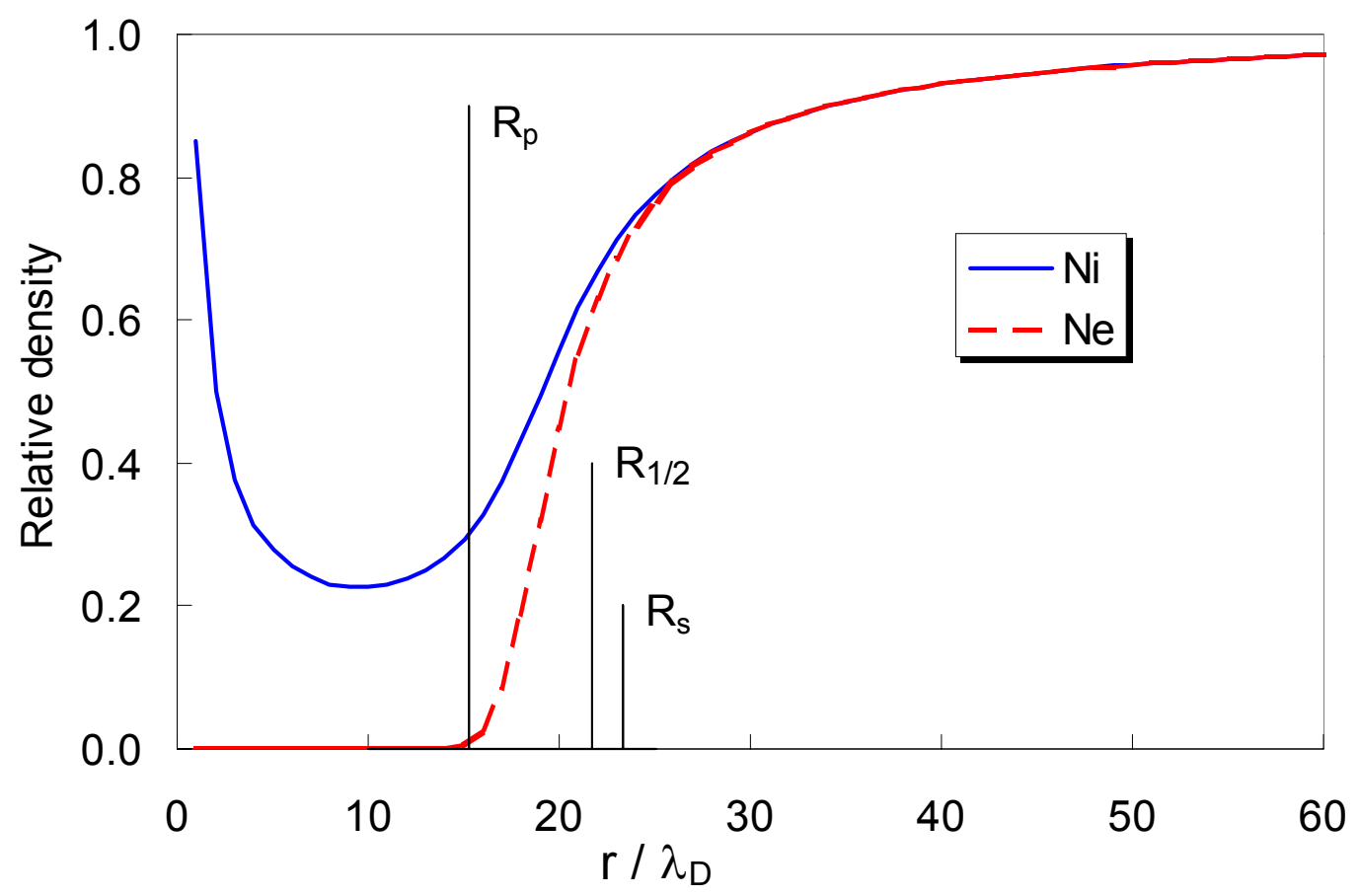

Fig. 16. ABR solution for the ion ( - ) and electron $(----)$ densities in the sheath for the case $J=10$. The radius of a floating probe is shown by the line labeled $R_{\mathrm{p}}$. Similarly, $R_{1 / 2}$ is the radius where $\eta=1 / 2$, and $R_{\mathrm{s}}$ is the effective sheath radius for this case. For probes at higher $\eta_{\mathrm{p}}$, the line $R_{\mathrm{p}}$ will move to the left for fixed $J$. 